\title{
Phototransduction, Dark Adaptation, and Rhodopsin Regeneration The Proctor Lecture
}

\author{
Trevor D. Lamb ${ }^{*, 1}$ and Edward N. Pugh, Jr $r^{*, 2}$
}

$\mathrm{T}^{\mathrm{h}}$ his article is divided into two sections. The first outlines the molecular events that underlie the onset of phototransduction, the photoreceptor's response to light, and provides a quantitative model of its kinetics and amplification. Light responses measured both with single-cell techniques and with the electroretinogram (ERG) are compared with the predictions of the model, and an account is given of the differences in gain between amphibian and mammalian rods. The second section turns to recovery of the overall visual system after exposure of the eye to very intense "bleaching" exposures, and describes the cellular and molecular mechanisms involved in the regeneration of rhodopsin. A model is presented for the delivery of 11-cis retinal to opsin in the bleached rods, which accounts for the kinetics of psychophysical dark adaptation and rhodopsin regeneration, both in normal subjects and in a range of disease states.

The slide presentation of the 2006 Proctor Lecture is available online as a PowerPoint file at http://www.iovs.org/cgi/ content/full/47/12/5138/DC1

\section{Phototransduction}

The transduction of light into a neural signal takes place in the outer segment of a retinal rod or cone photoreceptor (Fig. 1). Here, we concentrate on the rods, which in the human retina outnumber cones by approximately 20:1 overall. The outer segment of the rod contains an enormous area of lipid bilayer membrane, arranged as flattened "discs," and this membrane is densely packed with the photopigment rhodopsin.

Rhodopsin is a member of the superfamily of seven-helix, G-protein-coupled receptor proteins (GPCRs). In contrast to chemosensing GPCRs, rhodopsin has its ligand, the light-absorbing "chromophore" retinaldehyde, prebound. The bound form, 11-cis retinal, acts as a powerful antagonist, holding rhodopsin in its completely inactive state. Absorption of a photon of light isomerizes the chromophore to a second form, the all-trans configuration, which acts as a powerful agonist

From the ${ }^{1}$ Division of Neuroscience, John Curtin School of Medical Research, and ARC Centre of Excellence in Vision Science, The Australian National University, Canberra, Australia, and ${ }^{2}$ F. M. Kirby Center for Molecular Ophthalmology, Department of Ophthalmology, University of Pennsylvania, Philadelphia, Pennsylvania.

Supported by Australian Research Council Grants FF0344672 and CE0561903, National Eye Institute Grant EY02660, and Research to Prevent Blindness.

Disclosure: T.D. Lamb, None; E.N. Pugh, Jr, None

"Each of the following is a corresponding author: Trevor D. Lamb, Division of Neuroscience, John Curtin School of Medical Research, and ARC Centre of Excellence in Vision Science, The Australian National University, Canberra, ACT 0200, Australia; trevor.lamb@anu.edu.au. Edward N. Pugh, Jr, F. M. Kirby Center for Molecular Ophthalmology, Department of Ophthalmology, School of Medicine, University of Pennsylvania, Philadelphia, PA 19104-6096; pugh@mail.med.upenn.edu.

that rapidly triggers a conformational change in the protein, activating rhodopsin as an enzyme. In this first section, we will consider the mechanisms, the kinetics, and the gain of the rod's biochemical and electrical response to light absorption.

The conformational change triggered by light causes rhodopsin's absorption spectrum to shift into the UV, so that the pigment loses its visible color and is said to "bleach." Long after signaling is complete, the Schiff-base bond that attaches the all-trans retinal is hydrolyzed, permitting the retinoid to dissociate from the apo-protein, opsin. In the second section, we will analyze the regeneration of opsin to rhodopsin via a source of 11-cis retinal in the retinal pigment epithelium (RPE), and we will consider the consequences that the relatively slow kinetics of regeneration have for dark adaptation of the overall visual system. In this connection, we will discuss the biochemical reactions required to replenish the source of 11-cis retinoid from the released all-trans retinoid.

\section{The G-protein Cascade of Phototransduction}

G-protein cascades provide amplified signaling in a wide variety of cellular systems, in the great majority of cases as chemosensors-for example, in hormonal and synaptic signaling, as well as in olfactory reception. The molecular mechanism has been particularly well studied in photoreceptors, and the $\mathrm{G}^{-}$ protein cascade of phototransduction (Fig. 2) can serve as a model for other systems, particularly for their quantitative properties (for reviews of the phototransduction cascade, see Refs. 1-5).

In addition to rhodopsin (R), which is packed into the disc membrane at a density of approximately 25,000 molecules $\mu \mathrm{m}^{-2}$, three other species of protein play essential roles in activation of the response to light: (1) the heterotrimeric Gprotein $(G)$, termed transducin in rods, present at approximately 1:10 relative to rhodopsin; (2) the cyclic nucleotide phosphodiesterase (PDE), which hydrolyzes cyclic GMP, the diffusible cytoplasmic messenger, present at approximately 1:100 relative to rhodopsin; and (3) the cyclic nucleotide-gated channels (CNGCs) of the plasma membrane, which control the flow of electrical current into the outer segment.

Activation of the cascade can be broken down into the five steps indicated in Figure 2. The first three steps involve proteins associated with the disc membrane (rhodopsin, which is integral, and the G-protein and PDE, which are attached by acyl groups), whereas the final two steps involve the cytoplasmic messenger, cyclic GMP, and its action at the plasma membrane.

Step 1: Activation of Rhodopsin. First, a photon of light is absorbed by any one of the $\sim 10^{8}$ rhodopsin molecules in the outer segment, isomerizing the chromophore to its all-trans configuration and thereby activating that rhodopsin molecule to a form denoted as $\mathrm{R}^{*}$.

Step 2: Activation of the G-protein. All the proteins are mobile in (or at the surface of) the disc membrane, so that they diffuse laterally; consequently, the activated $R^{*}$ and inactive $G$ 

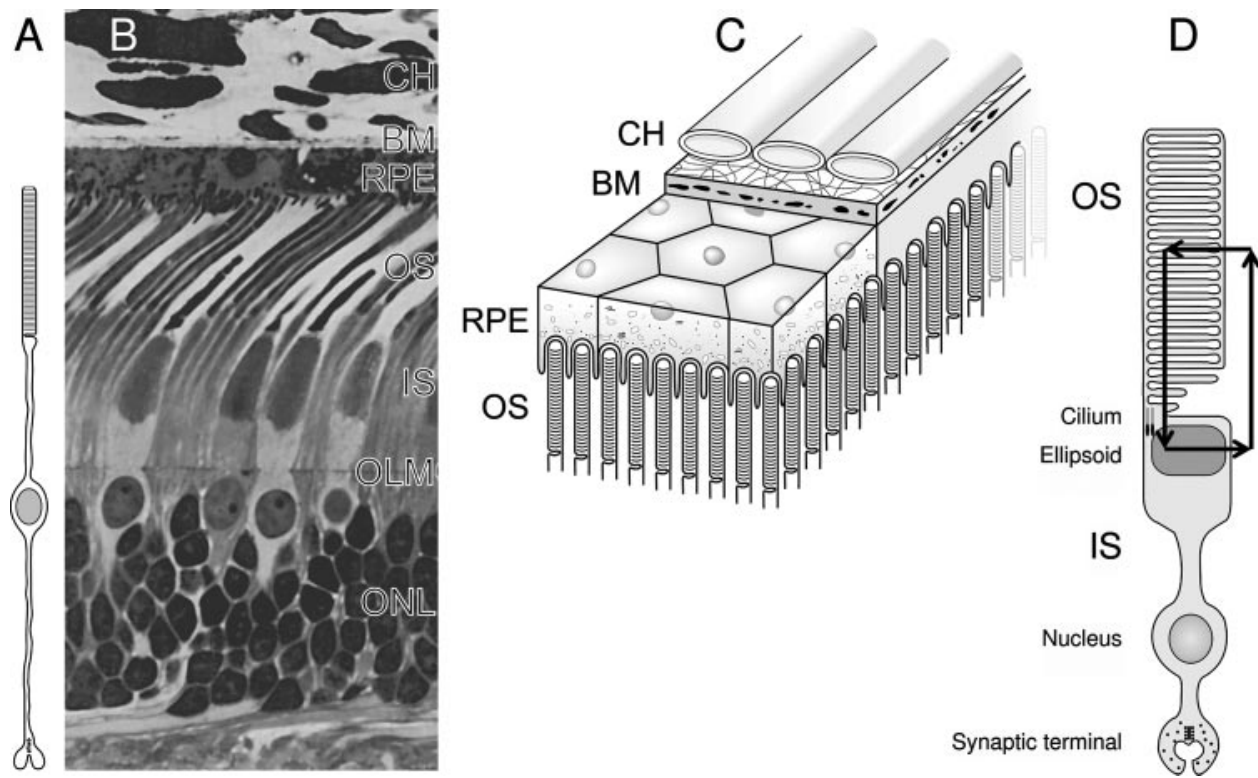

Figure 1. Anatomical features of the outer retina, retinal pigment epithelium, and photoreceptors. (A) Schematic of mammalian rod photoreceptor, illustrating disposition in relation to (B). (B) Light micrograph of the parafoveal region of rhesus monkey choroid, RPE, and distal retina. (C) Cutaway schematic of choriocapillaris, Bruch's membrane, retinal pigment epithelial cells, and tips of the rod outer segments. (D) Schematic of an amphibian rod, showing the circulating current. $\mathrm{CH}$, choriocapillaris; $\mathrm{BM}$, Bruch's membrane; RPE, retinal pigment epithelium; OS, outer segments; IS, inner segments; OLM, outer limiting membrane; ONL, outer nuclear layer. (A) Reproduced from Lamb TD, Pugh EN Jr. A quantitative account of the activation steps involved in phototransduction in amphibian photoreceptors. J Physiol. 1992;449: 719-758. (B) Reprinted with permission from Elsevier from Fisher SK, Anderson DH, Erickson PA, Guérin CJ, Lewis GP, Linberg KA. Light and electron microscopy of vertebrate photoreceptors. In: Hargrave PA, ed. Methods in Neurosciences 15, Photoreceptor cells. 1993:3-36. (C) Reprinted from Progress in Retinal and Eye Research 23, Lamb TD, Pugh EN Jr. Dark adaptation and the retinoid cycle of vision, 307-380, Copyright 2004, with permission from Elsevier. (D) Modified from Burns ME, Lamb TD. Visual transduction by rod and cone photoreceptors. In: Chalupa LM, Werner JS, eds. The Visual Neurosciences. Cambridge, MA: MIT Press; 1993:215-233.
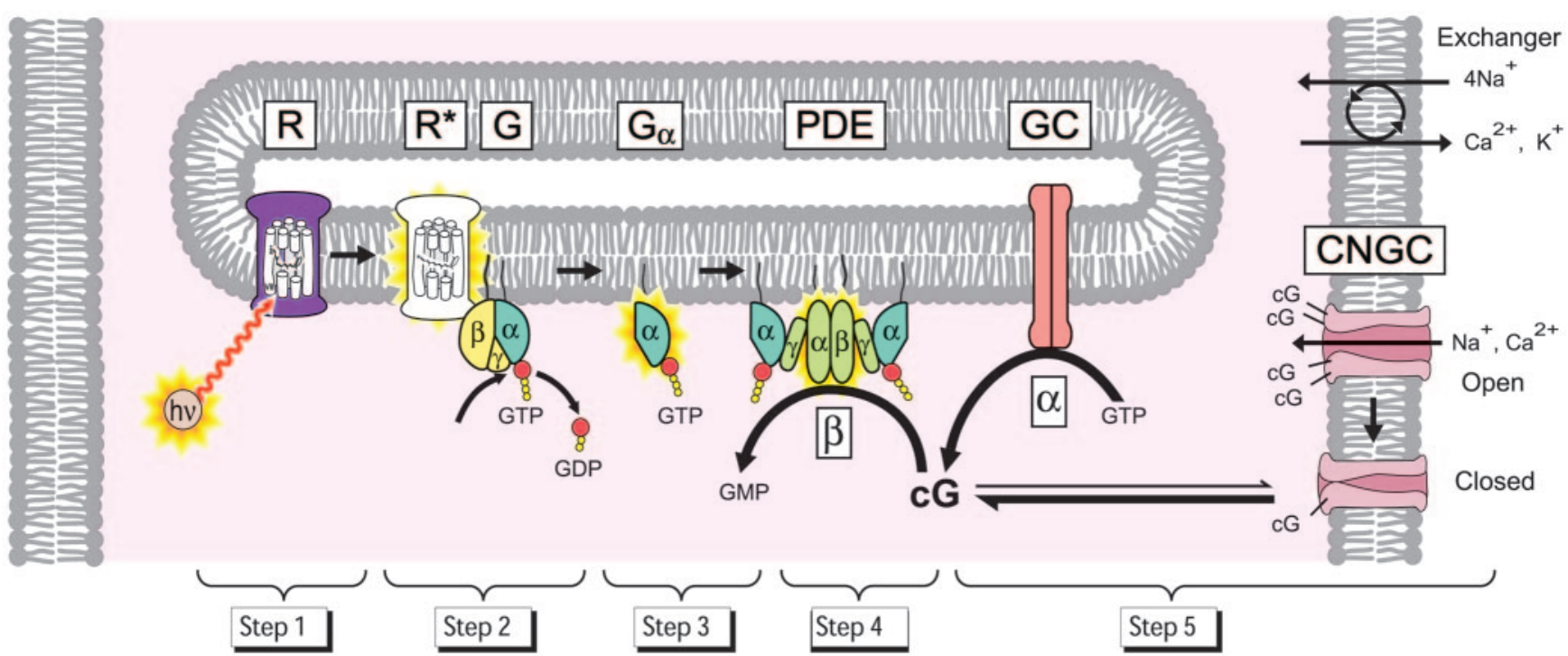

FiguRE 2. The G-protein cascade of phototransduction. After absorption of a photon $(\mathrm{h} \nu)$, the activated rhodopsin ( $\left.\mathrm{R}^{*}\right)$ repeatedly contacts molecules of the heterotrimeric G protein $(G)$, catalyzing the exchange of GDP for GTP, producing the active form G $\alpha$-GTP (i.e., G*). Two G*s bind to the inhibitory $\gamma$ subunits of the phosphodiesterase (PDE), thereby activating one or both (illustrated) of the corresponding $\alpha$ and $\beta$ catalytic subunits, which then catalyze the hydrolysis of cyclic GMP (cG). The consequent reduction in cytoplasmic concentration of cGMP leads to closure of the cyclic nucleotide gated channels and blockage of the inward flux of $\mathrm{Na}^{+}$and $\mathrm{Ca}^{2+}$, thereby reducing the circulating electrical current. The $\mathrm{Na}^{+} / \mathrm{Ca}^{2+}, \mathrm{K}^{+}$exchanger is not directly involved in activation; however, during the light response, it continues to pump Ca ${ }^{2+}$ out, so that the cytoplasmic $\mathrm{Ca}^{2+}$ concentration declines, aiding in recovery of the response by stimulating guanylyl cyclase (GC) activity, and other "calciumfeedback" mechanisms (reviewed in Refs. 3,8). 
come into contact, and bind transiently. In this $R^{*}-G$ state, a molecule of GDP that had been bound to the G-protein $\alpha$-subunit $\left(G_{\alpha}\right)$ is released, allowing a molecule of GTP from the cytoplasm to bind in its place. This process, called nucleotide exchange, activates the G-protein (to G-GTP), which then dissociates from $R^{*}$. Crucially, the activated rhodopsin $\left(R^{*}\right)$ has not been altered in any way by this interaction, and so it can contact another molecule of G-protein by diffusion and trigger nucleotide exchange again. This process can repeat indefinitely while $\mathrm{R}^{*}$ remains active, so that $\mathrm{R}^{*}$ can be regarded as an enzyme that catalytically activates numerous molecules of $\mathrm{G}^{-}$ protein.

Step 3: Activation of the PDE. When each activated Gprotein separates from $\mathrm{R}^{*}$, after nucleotide exchange, the message is carried forward by the $\alpha$-subunit, now in the form $\mathrm{G}_{\alpha}-\mathrm{GTP}$, denoted here as $\mathrm{G}^{*}$. As a result of lateral diffusion, the $\mathrm{G}^{*}$ comes into contact with the PDE and binds to one of its two regulatory $\gamma$-subunits, thereby partly activating the PDE to a form that we denote $E^{*}$. Subsequently, a second $\mathrm{G}^{*}$ can bind to the second $\gamma$-subunit, to fully activate the PDE. Unlike step 2, this activation has no amplification: A single $G^{*}$ can activate at most a single PDE catalytic subunit.

Step 4: Hydrolysis of Cyclic GMP. In the dark resting state, there is a steady balance between the synthesis of cyclic GMP by guanylyl cyclase (GC) and the slow hydrolysis of cyclic GMP by residual PDE activity, and hence there exists a steady, moderate cytoplasmic concentration (a few micromolar) of cyclic GMP. In response to illumination, the disc proteins are activated (steps 1-3), and the resultant activation of $E^{*}$ increases the rate of hydrolysis of cyclic GMP, so that the cyclic GMP concentration drops.

Step 5: Closure of Ion Channels. At its resting concentration in the dark, cyclic GMP binds to, and holds open, a proportion of the ion channels in the cell's plasma membrane. Although this proportion is quite small (typically just a few percent), the number of open channels is sufficient to conduct the substantial cation current (tens of pA) that flows into the outer segment in darkness. When the concentration of cyclic GMP drops in the light, cyclic GMP unbinds from the channels, causing the channels to close, and thereby generating the cell's electrical response-a reduction in the circulating current and a consequent hyperpolarization.

\section{Predicted Kinetics and Amplification}

Our goal was to predict the kinetics and amplification of this system, and a summary of our predictions ${ }^{1}$ is illustrated in Figure 3. To simplify the analysis, we ignored inactivation reactions, ${ }^{1,2}$ with the consequence that our subsequent comparison of theory with experiment needs to be restricted to relatively early times, before shut-off reactions come into play.

$\mathbf{R}^{*}$ Kinetics. In response to a single photon captured at time 0 , a single $R^{*}$ will be activated, after a very short (millisecond) delay due to internal rearrangements in the protein. And because inactivation is being ignored, the $\mathrm{R}^{*}$ will remain present, so that $R^{*}(t)$ will follow a step function of time (Fig. 3A).

$G^{*}$ Kinetics. While the single $R^{*}$ is present, $G^{*} s$ will be activated at a constant rate, and so the concentration $G^{*}(t)$ will ramp with time (Fig. 3B). The rate of activation has been estimated as around $125 \mathrm{G}^{*} \mathrm{~s}^{-1}$ per $\mathrm{R}^{*}$ for amphibian rods at room temperature ${ }^{9}$; in mammalian rods at body temperature, the rate is likely to be approximately three times higherapproximately $400 \mathrm{G}^{*} \mathrm{~s}^{-1}$ per $\mathrm{R}^{*}$.

$\mathbf{E}^{*}$ Kinetics. For activation of the PDE, the theoretical analysis is more complicated, because multiple molecules of $G^{*}$ are diffusing in a sea of PDEs. Nevertheless, it is possible to show $^{1}$ that, to a reasonable approximation, the $\mathrm{E}^{*}$ concentra-
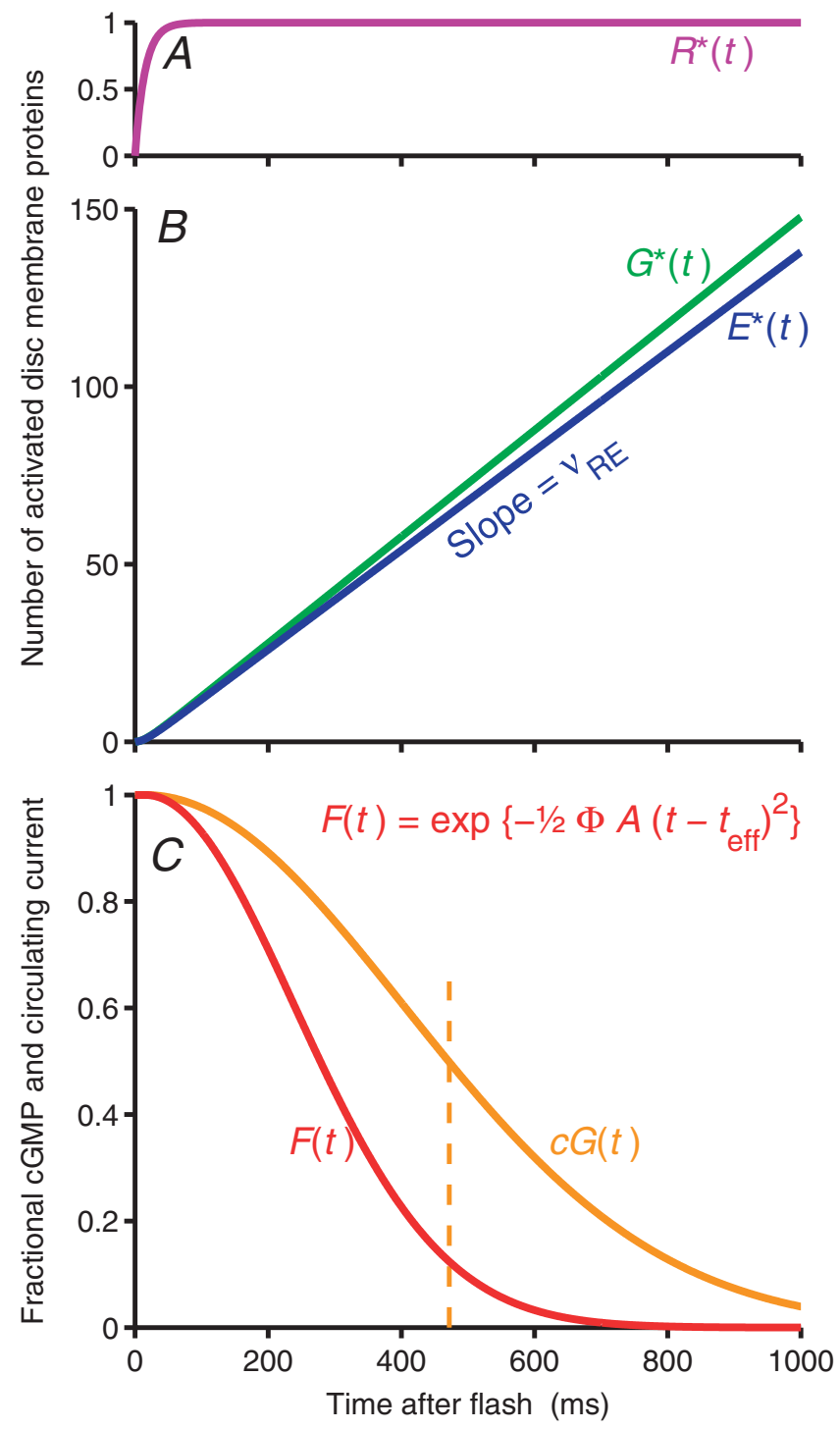

FIGURE 3. Predicted time-course of the amphibian rod's electrical response, when inactivation reactions are ignored. (A) After photoisomerization at time 0 , a single $\mathrm{R}^{*}$ is activated with a slight delay. (B) The quantities $G^{*}(t)$ and $E^{*}(t)$ of activated G-protein and PDE increase linearly with time after $\mathrm{R}^{*}$ activation; in both cases the slope is estimated to be around 100 to $150 \mathrm{~s}^{-1}$ per $\mathrm{R}^{*}$ at room temperature. (C) Activation of $\mathrm{E}^{*}$ causes the cyclic GMP concentration to decline. Consequently, cyclic nucleotide-gated channels close, and the fractional circulating current $F(t)$ declines according to equation 1 . Responses in (C) are shown for a flash delivering $\Phi=200$ photoisomerizations, with amplification constant $A=0.10 \mathrm{~s}^{-2}$. Dashed vertical line indicates when $c G=1 / 2$; at this time (and with $n_{\mathrm{cG}}=3$ ), the fraction of channels open is $F=1 / 8$.

tion should also ramp with time (Fig. 3B), though with a slightly lower slope than for $G^{*}$, because the coupling efficiency may be less than $100 \%$. We denote the slope of the $E^{*}(t)$ curve in Figure $3 \mathrm{~B}$ as $\nu_{\mathrm{RE}}$, signifying the rate at which a single $\mathrm{R}^{*}$ triggers activation of $\mathrm{E}^{*}$ subunits.

Cyclic GMP Kinetics. The hydrolytic activity of the phosphodiesterase can be expressed as the product of the number $E^{*}(t)$ of PDE subunits and the catalytic efficacy per subunit, denoted $\beta_{\text {sub }}$. The ramping of $E^{*}(t)$ therefore causes hydrolysis of cyclic GMP to occur at an ever accelerating rate, with the consequence that the cyclic GMP concentration $c G(t)$ begins declining parabolically with time (i.e., with an initial shape 
proportional to $t^{2}$ ). At later times, when relatively little cyclic GMP remains available to be hydrolyzed, the slope becomes less steep.

Closure of Ion Channels. When present in the cytoplasm, cyclic GMP binds to and opens plasma membrane ion channels, and it does so with cooperativity described by the Hill equation. At the low concentrations experienced under normal conditions, the fraction of channels open approximates a power-law relation, with exponent $n \approx 2-3$. In the case of $n=3$, the fraction of open channels, $F(t)$, would vary as the cube of the concentration of cyclic GMP (i.e., $\left.F(t) \propto c G(t)^{3}\right)$. Hence, at a time when $c G(t)$ had dropped to $1 / 2$ (dashed vertical line in Fig. 3C), the fraction of channels remaining open would have dropped to approximately $1 / 8$. And because the outer segment current depends only very weakly on intracellular voltage, ${ }^{10}$ $F(t)$ also represents the fractional circulating current.

Detailed Analysis. When we undertook a detailed analysis of the rate equations for the cascade, ${ }^{1,2}$ we were able to derive a simple equation for predicting the fractional circulating current $F(t)$ in response to a brief flash of light. We showed that, to a good approximation, the response should be described by a "delayed Gaussian" function of time

$$
F(t)=\exp \left\{-1 / 2 \Phi A\left(t-t_{\mathrm{eff}}\right)^{2}\right\}, \text { for } t>t_{\mathrm{eff}},
$$

where $\Phi$ is the number of photoisomerizations (the flash intensity), $A$ is a fixed parameter termed the "amplification constant" of transduction, and $t_{\text {eff }}$ is an "effective delay time" that lumps together a number of short delays in transduction.

Two important points should be made about equation 1. First, it provides what is essentially a single-parameter description of the onset phase of the light response. Thus, apart from the short delay term, $t_{\text {eff }}$, there is only one parameter, $A$, involved in describing the onset of the electrical response to any arbitrary flash intensity $\Phi$. Second, we were able to show that the overall amplification constant $A$ of the electrical response can be expressed as the product of individual "gain factors" contributed by the underlying biochemical stages, as

$$
A=\nu_{\mathrm{RE}} \beta_{\mathrm{sub}} n_{\mathrm{cG}}
$$

Here, $\nu_{\mathrm{RE}}$ is the rate of $\mathrm{E}^{*}$ activation in response to a single photoisomerization; $\beta_{\text {sub }}$ is the rate constant of cGMP hydrolysis by a single $E^{*}$ subunit, averaged throughout the outer segment volume; and $n_{\mathrm{CG}}$ is the Hill coefficient of channel opening cooperativity by cyclic GMP.

Furthermore, the PDE's hydrolytic efficacy, $\beta_{\text {sub }}$, could be expressed as

$$
\beta_{\mathrm{sub}}=\frac{1 / 2 \boldsymbol{k}_{\mathrm{cat}} / K_{m}}{N_{\mathrm{Av}} V_{\mathrm{cyto}} \boldsymbol{B}_{\mathrm{cG}}},
$$

where $k_{\text {cat }}$ and $K_{\mathrm{m}}$ are the catalytic activity and $K_{\mathrm{m}}$ of the fully-activated PDE, $N_{\mathrm{Av}}$ is Avogadro's number, $V_{\text {cyto }}$ is the cytoplasmic volume of the outer segment, and $B_{\mathrm{cG}}$ is the cytoplasmic buffering power for cGMP. (The factor $1 / 2$ converts a $k_{\text {cat }}$ value for the holoenzyme into a $\beta_{\text {sub }}$ value for an $\mathrm{E}^{*}$ subunit.)

The two subsidiary equations 2 and 3 reveal the remarkable fact that all the physical and biochemical parameters governing the activation of the cascade illustrated in Figure 2 collapse into a single parameter: the amplification constant $A$ of the cell's electrical response to light. Accordingly, this analysis provides a simple theoretical expression for predicting the flash response kinetics (in the absence of inactivation reac- tions), together with an account of the photoreceptor's overall amplification in terms of the gains of the underlying reactions.

Finally, we note that at early times the expression in \{\} in equation 1 is small, and so the normalized response, $R(t)=1-$ $F(t)$, is predicted to approximate a parabolic rise, according to

$$
R(t) \approx 1 / 2 \Phi A\left(t-t_{\mathrm{eff}}\right)^{2}
$$

\section{Comparison with Experiment}

The predictions of our model for the onset phase of the light response are compared with experiment in Figure 4. Figures $4 \mathrm{~A}$ and $4 \mathrm{~B}$ show single-cell recordings made with the suction pipette technique illustrated in Figure 5, whereas Figures 4C and $4 \mathrm{D}$ show ERG a-wave recordings. In all cases the stimuli were light flashes centered at time 0 , with measured intensities spanning a range of at least 100-fold. In each panel, the theory traces (red) provide a good description of the activation phase of the entire family of measured responses (black), across the whole range of flash intensities.

Figure $4 \mathrm{~A}$ illustrates suction pipette responses from a salamander rod. ${ }^{11}$ The red traces plot the predictions of equation 1, using the measured light intensities $\Phi$ (spanning a 200 -fold range), together with $A=0.065 \mathrm{~s}^{-2}$ and $t_{\text {eff }}=20 \mathrm{~ms}$, and they provide a good description of the measured responses. In this panel, the theory traces remain close to the recorded traces for longer than would normally be expected, because the activation of GC by lowered intracellular calcium (and possibly other "calcium feedback" mechanisms), which normally serve to speed the recovery of the circulating current, have been slowed. For this experiment, the calcium buffer BAPTA had been incorporated into the rod's cytoplasm (using a whole-cell patch pipette, as illustrated in the micrograph in Fig. 5), with the consequence that the slowing of the lightinduced changes in $\mathrm{Ca}^{2+}$ concentration retarded the onset of such recovery reactions.

Figure $4 \mathrm{~B}$ illustrates suction pipette responses from a human rod. ${ }^{12}$ Such recordings are extremely difficult, in part because of the very small diameter of mammalian rods, but also because of the issues involved in working with living human tissues postmortem and the need to regenerate (using 11-cis retinal) rhodopsin that was bleached under operating theater lights. Again the red traces, which plot the predictions of equation 1 for intensities spanning a 100-fold range, provide a good description of the measured responses, at least out to 60 ms after the flash. For this human rod, the parameters used were $A=4 \mathrm{~s}^{-2}$ and $t_{\text {eff }}=2.2 \mathrm{~ms}$.

It has long been known that the ERG a-wave response recorded from a dark-adapted eye arises primarily from the electrical activity of rod photoreceptors ${ }^{16}$ and indeed that the $a$-wave monitors the rod's circulating electrical current. ${ }^{17}$ As shown by the red traces in Figures $4 \mathrm{~A}$ and $4 \mathrm{C}$, the predictions of the model account accurately for the onset phase of the a-wave, over a wide range of intensities ( $>1800$-fold in each panel).

A notable feature of a-wave responses to bright flashes is the occurrence of an initial peak after some 5 to $10 \mathrm{~ms}$, followed by a rapid sag reminiscent of intracellular voltage responses of rods. In the past, it has usually been presumed that this sag represents an early manifestation of postreceptoral b-wave signals. However, recent evidence suggests instead that this "a-wave recovery" phase arises from intrinsic photoreceptor activity, probably due to an $I_{\mathrm{h}}$ current in the rod's inner segment. ${ }^{18,19}$ In any case, the red traces predicted by the simplified theory are expected to be applicable only until the intervention of any shut-off or recovery events.

Also of note in the two ERG panels (Figs. 4C, 4D) is the very short time scale over which the responses have been exam- 

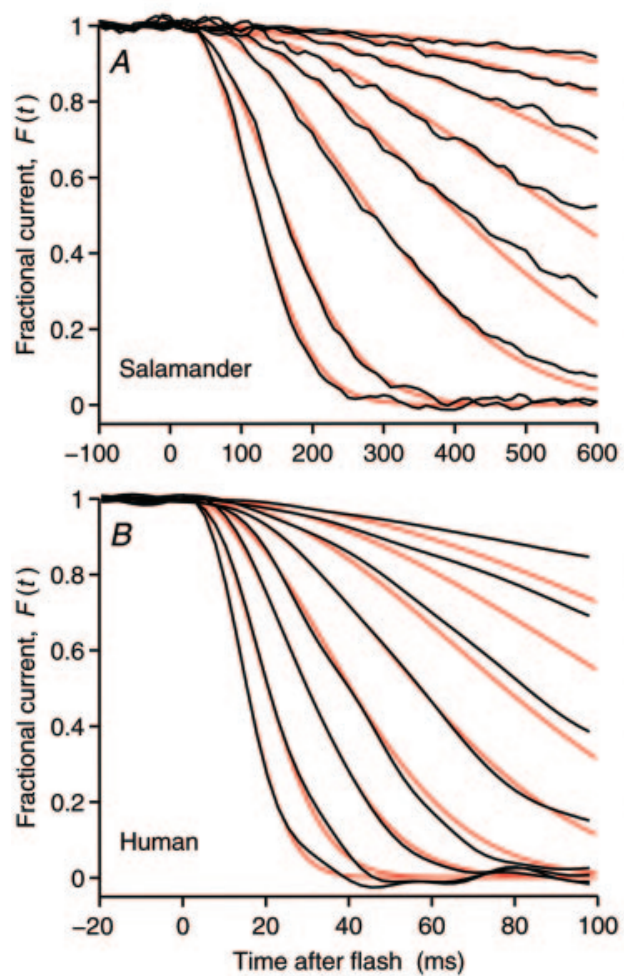

Scotopic ERG a-wave

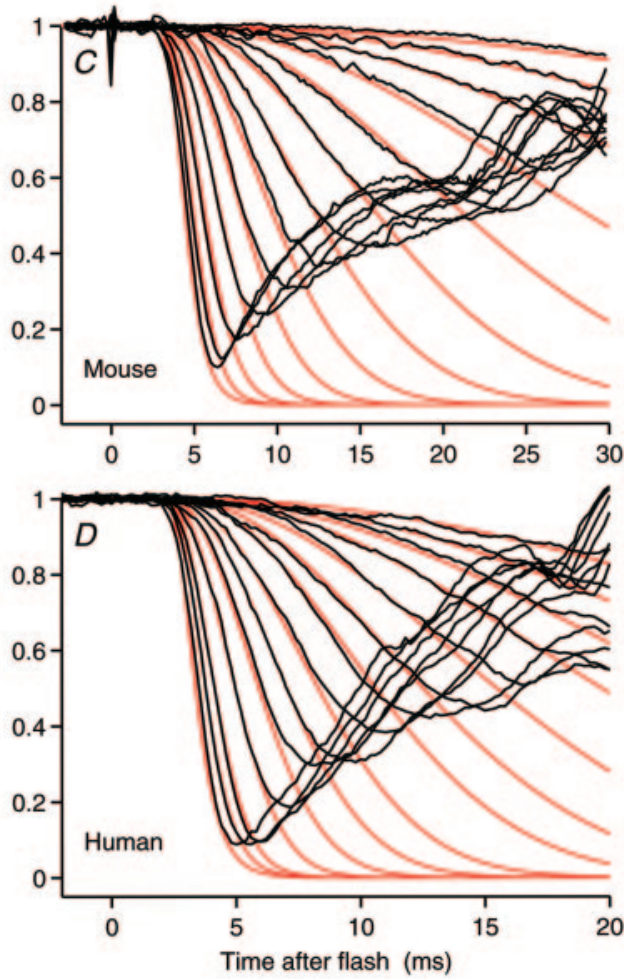

Figure 4. Comparison of prediction and experiment, for families of flash responses recorded using the suction pipette method (A, C) or the scotopic ERG $a$-wave $(\mathbf{B}, \mathbf{D})$. Black traces: experiment; red traces: plot of equation 1, using calculated number of photoisomerizations, $\Phi$, and fixed values of amplification constant $A$ and effective delay time $t_{\text {eff }}$. (A) Salamander rod after incorporation of the calcium buffer BAPTA (data from Figure 2B of Ref. 11); dark current, $-29 \mathrm{pA} ; A=0.065 \mathrm{~s}^{-2} ; t_{\mathrm{eff}}=$ $20 \mathrm{~ms}$. (B) Human rod (data from Figure 4 of Ref. 12); dark current, $-13.5 \mathrm{pA} ; A=4 \mathrm{~s}^{-2} ; t_{\mathrm{eff}}=2.2 \mathrm{~ms}$. (A, B) Time origin is middle of flash stimulus, after allowance for electronic filtering delay. (C) Mouse ERG a-waves (data from Figure 3 of Ref. 13); $a_{\max }=-500 \mu \mathrm{V}, A=5 \mathrm{~s}^{-2}, t_{\mathrm{eff}}$ $=2.6 \mathrm{~ms}, \tau_{\mathrm{m}}=0.8 \mathrm{~ms}$. (D) Human rod-isolated ERG $a$-waves (data from Figure $1 \mathrm{E}$ of Ref. 14 for subject TDL); $a_{\max }=-207 \mu \mathrm{V}, A=4.3 \mathrm{~s}^{-2}, t_{\mathrm{eff}}=$ $2 \mathrm{~ms}, \tau_{\mathrm{m}}=0.7 \mathrm{~ms}$. For the ERG traces, the predictions of equation 1 have been filtered by a time constant $\tau_{\mathrm{m}}$ representing the cell's capacitive time constant ${ }^{15}$; for the single-cell recordings, that time constant is lumped into the total delay time. The

values for $\Phi$, and hence for $A$, are based on the following intensity-conversion factors: (A) Salamander rod collecting area, $A_{\mathrm{c}}=20 \mu \mathrm{m}^{2} ;(\mathbf{B})$ human rod, $A_{\mathrm{c}}=0.85 \mu \mathrm{m}^{2}$ (reduced from the original value of $1.7 \mu \mathrm{m}^{2}$ on the basis that the diameter of the rod outer segment is likely to have been 1.4 $\mu \mathrm{m}$ rather than $2 \mu \mathrm{m}$ ); (C) mouse eye, rod collecting area at the cornea, $0.2 \mu \mathrm{m}^{2}$; (D) human eye, troland conversion factor $K=8.6$ isomerizations $\mathrm{s}^{-1} \mathrm{Td}^{-1}$.

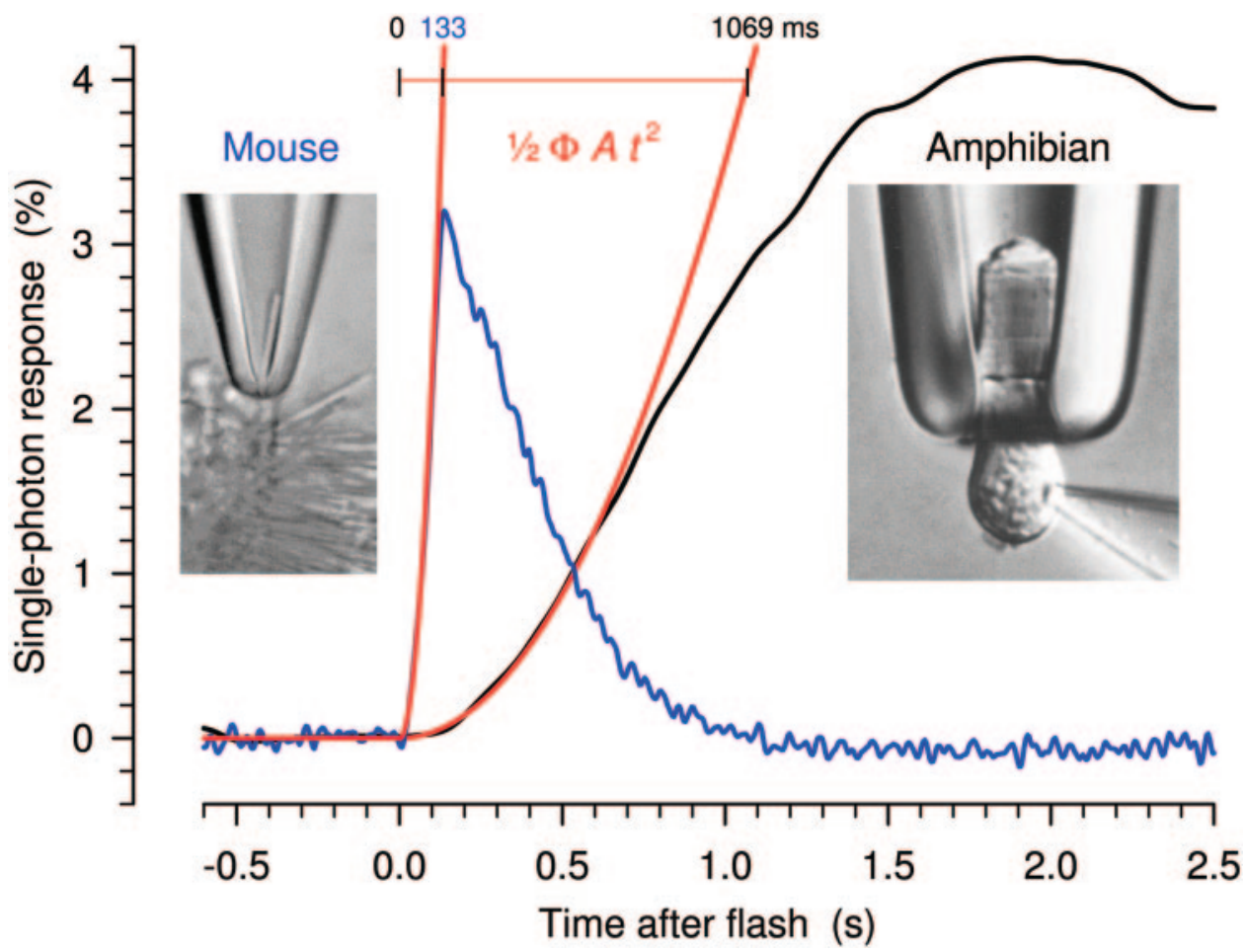

Figure 5. Comparison of singlephoton responses, amplification constants, and anatomy of mammalian and amphibian rods. Blue trace: mean single-photon response for 21 wild type (WT) mouse rods (from Figure 2B of Ref. 23); black trace: mean single-photon response for a toad rod (from Figure 1C of Ref. 24). Responses have been scaled as percentage of maximum response (mouse, $12.8 \mathrm{pA}$; toad, $42 \mathrm{pA}$ ). They have been shifted laterally, so that the midpoint of the test flash occurred at time 0 after allowance for the filter delay and the transduction delay; thus, the recorded traces have been adjusted for $t_{\text {eff. Red traces: }}$ plot of the expected parabolic early rise of equation $1, R(t) \approx 1 / 2 A t^{2}$, using $A=4.5 \mathrm{~s}^{-2}$ (mouse) and $A=$ $0.07 \mathrm{~s}^{-2}$ (toad). Horizontal red line: a specific example of the faster rise of the parabola with the higher amplification constant; thus, a level of $4 \%$ is crossed at 133 and $1069 \mathrm{~ms}$, respectively, where 1069/133= $\sqrt{ }(4.5 / 0.07)=\sim 8$-fold. Mouse rods were recorded at 34 to $37^{\circ} \mathrm{C}$, and low-pass Bessel filtered at $20 \mathrm{~Hz}$; toad rod was recorded at $\sim 22^{\circ} \mathrm{C}$ and digitally low-pass filtered at $2.5 \mathrm{~Hz}$. Insets: micrographs of a mouse $\operatorname{rod}^{25}$ and a salamander rod, ${ }^{26}$ at similar scales, to emphasize the difference in volume between mammalian and amphibian rod outer segments. For outer segments identical in all respects except diameter, the speed of the early parabolic rise of the single-photon response would be inversely proportional to outer segment diameter, because of the volume factor in the denominator of equation 3. Micrograph of mouse rod reproduced from Baylor DA, Burns ME. Control of rhodopsin activity in vision. Eye. 1998;12:521-525. (c) Royal College of Ophthalmologists. Micrograph of salamander rod reprinted from Lamb TD, Matthews HR, Torre V. Incorporation of calcium buffers into salamander retinal rods: a rejection of the calcium hypothesis of phototransduction. $J$ Physiol. $1986 ; 372: 315-349$. 
TABLE 1. Amplification Constants and Underlying Gain Factors in Amphibian and Mammalian Rods

\begin{tabular}{|c|c|c|c|c|c|c|c|c|}
\hline & & $\begin{array}{c}\text { Amplification Constant } \\
\underset{\left(s^{-2}\right)}{ }\end{array}$ & $=$ & $\begin{array}{c}\mathbf{E}^{*} \text { Rate } \\
\left(\text { per } \mathbf{R}^{*}\right) \\
\nu_{\mathbf{R E}} \\
\left(\mathbf{s}^{-1}\right)\end{array}$ & $x$ & $\begin{array}{c}\text { Hydrolysis Rate } \\
\left(\text { per } \mathbf{E}^{*}\right) \\
\boldsymbol{\beta}_{\text {sub }} \\
\left(\times 10^{-3} \mathbf{s}^{-1}\right)\end{array}$ & $x$ & $\begin{array}{c}\text { Hill Coefficient } \\
n_{\mathrm{cG}}\end{array}$ \\
\hline Toad & $22^{\circ} \mathrm{C}$ & 0.1 & $=$ & 125 & $\times$ & 0.4 & $\times$ & 2 \\
\hline Mouse & $37^{\circ} \mathrm{C}$ & 8 & $=$ & 400 & $\times$ & 10 & $\times$ & 2 \\
\hline Ratio & & 80 & $=$ & 3.2 & $\times$ & 25 & & \\
\hline
\end{tabular}

Parameter values for rods of amphibians and mammals (exemplified by toad and mouse), are given in round numbers. The values of $\nu_{\mathrm{RE}}$ and $\beta_{\text {sub }}$ for toad are from Reference 9. The amplification constants are from References 2 and 21, see also Reference 22. These values correspond to a temperature factor of around 3.2-fold and a volume factor of around 25-fold between the rods of the two species.

ined. Such a short time scale is necessitated by the intrusion of nontransduction signals within approximately $30 \mathrm{~ms}$ of the flash. As a result, one must study flash intensities much higher than those typically used for single-cell recordings. In Figures $4 \mathrm{C}$ and $4 \mathrm{D}$, the brightest flashes used were $>200,000$ photoisomerizations per flash. For such fast responses, it becomes necessary to take into account the capacitance of the cell membrane. Hence, for the red traces in the ERG panels, the predictions of equation 1 have been filtered by a membrane time constant of $\tau_{\mathrm{m}} \approx 0.75 \mathrm{~ms}$. Such filtering can be performed either analytically ${ }^{15}$ or numerically.

Figure 4C illustrates ERG a-wave responses from the eye of an anesthetized mouse, ${ }^{13}$ for flash intensities ranging from approximately 50 to 200,000 isomerizations, whereas Figure 4D illustrates ERG a-wave responses from a human eye, ${ }^{14}$ for flashes from approximately 300 to 550,000 isomerizations. For these two panels, the values of amplification constant used were $A=5.0 \mathrm{~s}^{-2}$ (mouse rods) and $A=4.3 \mathrm{~s}^{-2}$ (human rods).

The conclusion of this analysis is that the family of waveforms predicted by equation 1 provides an accurate description of the time course of the onset phase of rod responses to light, over a wide range of flash intensities. Thus, the kinetics predicted by analysis of the biochemical cascade of reactions in Figure 2 are completely consistent with electrical responses measured in experiments. The quality of the fit in Figures $4 \mathrm{C}$ and $4 \mathrm{D}$ provides further evidence that the scotopic ERG a-wave does indeed arise from suppression of the rod photoreceptor circulating current.

\section{Amplification Constant: Comparison of Amphibian and Mammalian Rods}

From the parameters used for the red traces in Figure 4, it is clear that the amplification constant of mammalian rods is much higher than that of amphibian rods. Across different laboratories and different cells, the mean value of amplification constant for the large rod outer segments of amphibia recorded at room temperature is around $A=0.1 \mathrm{~s}^{-2}$, whereas for the small rod outer segments of mammals recorded at body temperature, the mean is around $A=8 \mathrm{~s}^{-2}$, a factor of around 80-fold higher (see, for example, Refs. 2,9,20-22).

Response Speed. The role of the amplification constant in determining the speed of the response is illustrated in Figure 5, which compares the averaged single-photon response from a toad rod (black trace) with that from wild-type mouse rods (blue trace). Such responses to single photons have the advantage that uncertainties in light calibrations and conversion factors are avoided. For a single photoisomerization $(\Phi=1)$, equation 4 gives the scaling of the early parabolic rise as $R(t) \approx$ $1 / 2 A\left(t-t_{\mathrm{eff}}\right),{ }^{2}$ as plotted by the red curves. For the illustrated responses, the values of amplification constant used were $A=$ $0.07 \mathrm{~s}^{-2}$ (toad rod) and $A=4.5 \mathrm{~s}^{-2}$ (mouse WT rods). The significance for the response speed is readily appreciated: in this case, the 64-fold higher value of $A$ for the mouse rod response permits it to achieve any specific level of fractional suppression of circulating current $\sqrt{ } 64=$ eightfold faster.

What causes the amplification constant to be almost two orders of magnitude higher in mammalian rods than in amphibian rods?

Role of Temperature. The answer lies partly in the temperature dependence of the reactions. As indicated in equation 2 , the overall amplification constant $A$ is the product of the underlying gain steps, and estimates of these parameters are set out in Table 1 . The channel cooperativity $n_{\mathrm{cG}}$ is unlikely to differ greatly between amphibian and mammalian rods. However, the rate of activation of $\mathrm{E}^{*}$ subunits per $\mathrm{R}^{*}, \nu_{\mathrm{RE}}$, is likely to be several times higher at body temperature $\left(37^{\circ} \mathrm{C}\right)$ in mammalian rods than at room temperature $\left(22^{\circ} \mathrm{C}\right)$ in amphibian rods, because the underlying reactions take place at the disc membrane and depend on its fluidity. Previously, we estimated that the temperature dependence alone could account for an approximately a threefold increase in $A$ over this temperature range. $^{2}$ Recent evidence has suggested that the temperature effect could be even higher, ${ }^{22}$ but has also shown subtle differences in temperature dependence between amphibian and mammalian rods that makes precise comparison difficult.

Role of Outer Segment Volume. A contribution much larger than that due to temperature is provided by differences in the PDE hydrolysis factor, $\beta_{\text {sub }}$, the parameter specifying the efficacy with which a single $E^{*}$ subunit is able to reduce the average cyclic GMP concentration over the entire outer segment cytoplasm. As shown by equation 3, $\beta_{\text {sub }}$ is inversely proportional to the cytoplasmic volume $V_{\text {cyto }}$ of the rod outer segment, so that the overall amplification constant is also inversely proportional to $V_{\text {cyto }}$. As illustrated in Figure 5, mammalian rod outer segments are far smaller than their amphibian counterparts, with a volume ratio of $\sim 25$-fold. Recent experiments comparing the activation kinetics of rat and toad rod responses under identical conditions have confirmed these findings, with a 25- to 30-fold ratio of amplification constant. ${ }^{22}$ The interpretation of the volume effect is straightforward: the hydrolytic rate constant, $E^{*} \beta_{\text {sub }}$, of a given number of $\mathrm{E}^{*}$ subunits is greater in the small mammalian rod than in the large amphibian rod, according to the ratio of the cytoplasmic volumes, so that the reduction in cyclic GMP concentration occurs correspondingly faster. Our analysis has also shown that this effect applies even at the level of the single photon response, ${ }^{1}$ as illustrated in Figure 5.

The product of the gain factors yields the amplification constant. Table 1 demonstrates the important prediction of equation 2: that the gain factors arising in the individual steps multiply together to provide the overall amplification constant. In summary, our analysis of the G-protein cascade of phototransduction accounts for the gain as well as the onset-phase 


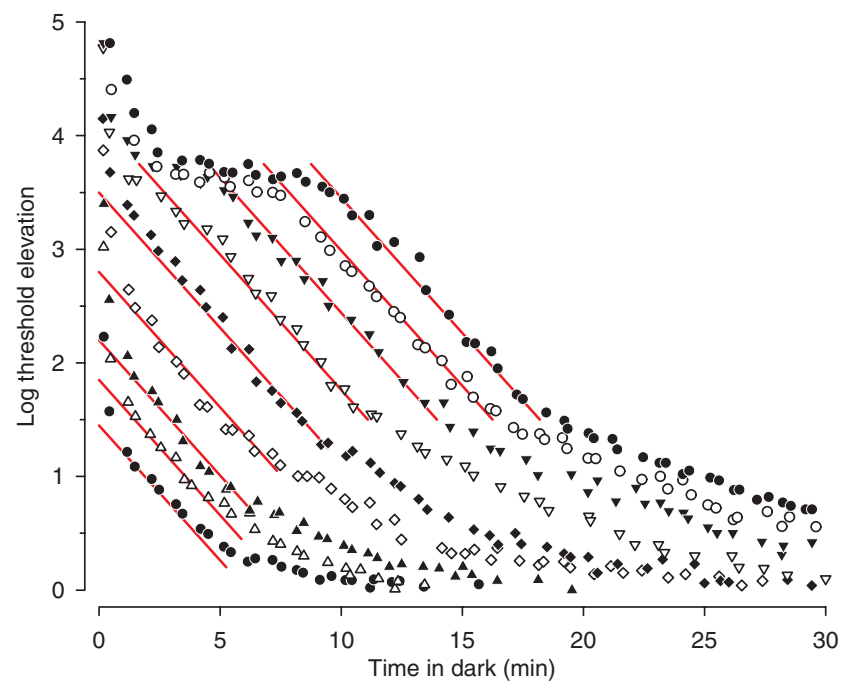

Figure 6. Human psychophysical dark adaptation. Recovery of log threshold elevation in a normal human observer is plotted as a function of time in darkness, after a wide range of bleaching exposures. Data from Reference 30 for subject ENP: The raw measurements have been averaged over bin widths of $30 \mathrm{~s}$ and over repetitions of the same bleach level. Symbols, from bottom left to top right, correspond to exposures ranging from 4.7 to $7.6 \mathrm{log}$ scotopic Td s, which were

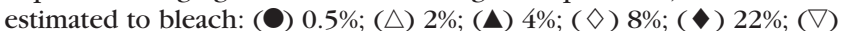
39\%; (V) 63\%; (○)s 86\%; (O) 98\% of the rhodopsin. Parallel red lines represent component "S2" with a negative slope of $\Psi_{\mathrm{S} 2}=0.24 \log _{10}$ units $\min ^{-1}$.

kinetics of the electrical response to flashes of light of arbitrary intensity, in both amphibian and mammalian rods.

\section{Dark Adaptation and Rhodopsin Regeneration}

After rhodopsin has been activated by light, it undergoes a series of inactivation reactions, passing through several intermediate forms (metarhodopsins and, after hydrolysis and release of the all-trans chromophore, opsin), before eventually being regenerated to the original state, rhodopsin, on binding of new 11-cis retinal.

It has been known for many decades that "dark adaptation," the slow recovery of visual sensitivity after exposure to a strong light, is somehow associated with the regeneration of visual pigment (reviewed in Ref. 7), yet the details of the relationship and the underlying cellular and molecular basis for it have remained unclear and controversial. In the 1960s it was hypothesized that unregenerated rhodopsin (i.e., opsin) elevates visual threshold during dark adaptation according to a powerful antilogarithmic relation, ${ }^{27,28}$ but subsequent results and analyses have revealed serious quantitative difficulties with this hypothesis. ${ }^{7,29}$

Here, we draw together published experimental results on dark adaptation and rhodopsin regeneration in the human eye with studies of the anatomy and biochemistry of retinoid processing. We present a cellular model for the delivery of retinoid to opsin in the bleached rod outer segments that is able to account for the observed kinetics of rhodopsin regeneration and of dark adaptation in the normal human eye, and that can also account for the slowed recovery that is found in a variety of disease conditions.

\section{Human Dark Adaptation}

The time course of psychophysical dark adaptation in a normal human subject (ENP) is plotted in Figure 6, from the data of Pugh. ${ }^{30}$ The elevation of threshold (in $\log _{10}$ units) for the detection of a visual stimulus is plotted against time in darkness, after exposures of nine different intensities, that were estimated to bleach from $0.5 \%$ to $98 \%$ of the rhodopsin. After a near-total bleach, recovery follows a classic biphasic form, with an early cone-mediated phase and a later rod-mediated phase. Initially, the threshold is elevated approximately $5 \log _{10}$ units (100,000-fold), but rapid recovery occurs over the first few minutes to a "cone plateau" approximately $3.7 \log _{10}$ units (5000-fold) above absolute threshold. After approximately 10 min (the "cone/rod break" time), the sensitivity of the rodmediated system is at last better than that of the cone system, and the threshold again begins declining with time.

The S2 Component of Recovery. Remarkably, the recovery of scotopic (rod-mediated) threshold exhibits a region of common slope across all the bleach levels, as indicated by the parallel red lines in Figure 6. This region is termed the "S2 component" of recovery ${ }^{29}$ and is crucial to our analysis of the underlying mechanisms. The slope of the red lines is $\Psi_{\mathrm{s} 2}=$ $0.24 \log _{10}$ unit $\mathrm{min}^{-1}$, and from analysis of these and other results, it appears that this value is a universal characteristic of dark adaptation recovery in normal (young adult) human eyes. $^{31}$

In interpreting this behavior, two facts should be borne in mind. First, a straight line in semilogarithmic coordinates corresponds to an exponential decay in linear coordinates, with the slope of $0.24 \log _{10}$ unit $\min ^{-1}$ corresponding to a time constant of $\tau_{\mathrm{s} 2}=1.8 \mathrm{~min}\left(=\log _{10}(\mathrm{e}) / 0.24\right)$. Second, it has been reported ever since the 1930 s that the after-effects of bleaching resemble, to a certain extent, the effects of real light. ${ }^{32}$

By combining these ideas with analysis of Pugh's data, ${ }^{30}$ Lamb suggested ${ }^{29}$ that the S2 region represents the exponential decay of a threshold-elevating substance produced by the bleach. Specifically, he proposed (1) that some chemical prod-

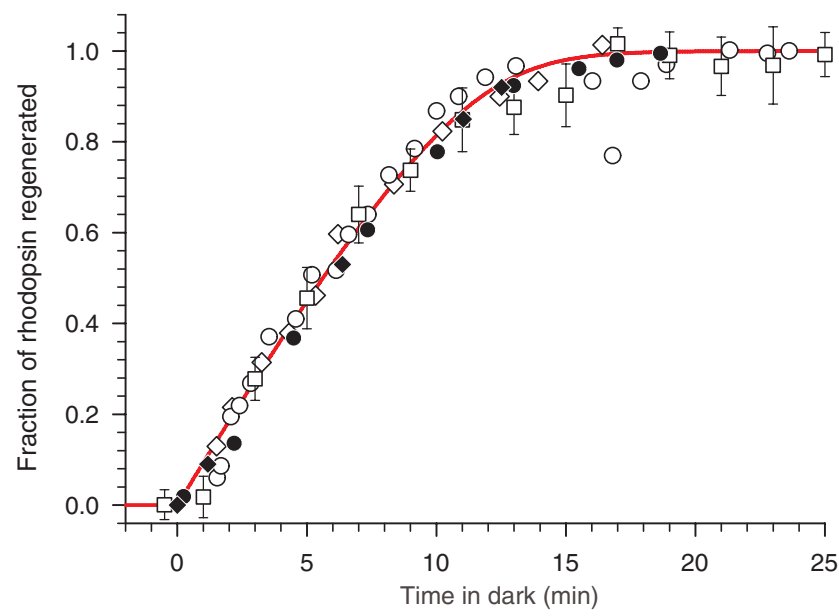

FIGURE 7. Regeneration of rhodopsin in the human retina, measured by retinal densitometry (open symbols), and estimated from psychophysical dark adaptation ( $)$ and from ERG $a$-wave recovery $(\diamond)$. Retinal densitometry measurements are: $(\bigcirc)$, from Figure 4 of Reference $41 ;(\diamond)$, from Figure 1 of Reference $42 ;(\square)$, from unpublished results of References 43, 44 mean \pm SEM over 2-min windows. Psychophysical recoveries: ( $\bigcirc$ ), values from Figure 9 of Reference 7 , extracted from the same data as presented in Figure 6. ERG a-wave recoveries: $(\checkmark)$, values from Figure 9 of Reference 7 , extracted from the data in Figure $8 \mathrm{~B}$ of Reference 45 . Curve plots MLP rate-limited kinetics predicted by equation 5, with $\nu=0.094 \min ^{-1}, K_{\mathrm{m}}=0.17$, and $B=1$ (i.e., for full bleaching). For $(\bigcirc)$ and $(\diamond)$, we estimate that the bleach levels were $87 \%$ and $89 \%$, and so the measurements have been shifted leftward by 1.4 and $1.2 \mathrm{~min}$, respectively $(0.13 / 0.094$ and $0.11 / 0.094)$, in order that they align with the predicted curve for a total bleach. 
uct was produced in direct proportion to the bleach, (2) that this substance generated a phenomenon equivalent to light, and (3) that the substance was removed by a reaction that was first-order for small bleaches. On this basis, the declining level of product would cause the "equivalent background intensity" to decline exponentially, in turn allowing the $\log _{10}$ visual threshold to decline as a straight line.

Hypothesis: Opsin Is the Substance Underlying S2. What might be the identity of this threshold-elevating substance underlying component $\mathrm{S} 2$ of human dark adaptation? In 1997 , we proposed this substance to be the opsin produced by bleaching (Lamb et al. IOVS 1997;38:ARVO Abstract 5263) and Reference 33. This proposal followed naturally from several in vitro studies that had shown first that opsin weakly activates the transduction cascade ${ }^{34,35}$ and second that recombination with 11-cis retinal eliminates opsin's activity. ${ }^{36,37}$ But before 1997 , it had not been hypothesized that opsin was the bleaching byproduct that underlies S2, nor had any kinetic evidence or analysis been presented that supported this identification. Indeed, the generally accepted view that rhodopsin's regeneration in the human eye followed exponential kinetics was strongly inconsistent with this hypothesis.

To examine the hypothesis, it was first necessary to reevaluate historical evidence concerning the time course of rhodopsin's regeneration and then to compare the kinetics of regeneration with the postbleach recovery of other features, such as the rod's circulating current (measured from the awave) and the threshold of the overall visual system (measured psychophysically). We begin with the kinetics of regeneration of rhodopsin.

\section{Regeneration of Rhodopsin in the Living Human Eye}

The technique of fundus reflection densitometry was developed in the 1950s as a means of measuring the levels of visual pigment in the living eye. ${ }^{38,39}$ A detailed analysis of the light paths likely to be involved in reflection densitometry measurements has been presented more recently. ${ }^{40}$

Measurements of the recovery of rhodopsin density after extensive bleaching are plotted as open symbols in Figure 7 , using data from three studies in the literature. ${ }^{41-44}$ For one of these studies (open squares) the bleach was total, but for the other two we estimate that the bleaching level was around $87 \%$ and $89 \%$. To compensate, those points (open circles, open diamonds) were shifted leftward by small amounts (13\% or $11 \%$ divided by the normalized regeneration rate). In addition, the filled symbols in Figure 7 plot estimates of rhodopsin regeneration obtained from the psychophysical dark adaptation experiment in Figure 6 (filled circles) and from ERG $a$-wave recoveries after bleaching (filled diamonds) (see legend to Figure 6).

Regeneration Follows Rate-Limited Kinetics. The form of recovery is not exponential, and instead the points can be quite well described by a straight line, up until approximately $70 \%$ recovery. Behavior of this kind has been well-documented in a variety of other mammalian species, ${ }^{39,46,47}$ but before our recent analyses, ${ }^{7,48}$ it had not been thought to occur in the normal human eye. The smooth curve in Figure 7 plots the prediction of a "rate-limited" model of rhodopsin regeneration $^{7,48}$ that provides a reasonable description of the data. The foundations for this model will be described once we have considered the anatomical and biochemical basis of retinoid processing in the human eye.

Before leaving the topic of pigment regeneration, we point out that in the late stages of recovery, when the levels of metarhodopsin product can be ignored, the quantity of opsin remaining is given by the complement of the rhodopsin regeneration curve: $O p s(t)=1-R h(t)$ in the normalized units of
Figure 7. Thus, the final exponential tail of rhodopsin regeneration corresponds to an exponential decline in the remaining level of opsin in the outer segment.

\section{Anatomy and Biochemistry of Retinoid Processing in the Eye}

The synthesis of fresh 11-cis retinal for delivery to opsin in the rods occurs in the retinal pigment epithelium (RPE) that lies just distal to the outer segments (Fig. 1). The RPE forms part of the blood-brain barrier, shielding the retina from the choroidal circulation, and it performs many other important functions, ${ }^{49}$ including active transport of metabolites, phagocytosis of the aged tips of photoreceptor outer segments, and absorption of light that manages to pass beyond the photoreceptors, in addition to synthesis of 11-cis retinal. In the periphery of the human retina, each RPE cell contacts a single cone photoreceptor and around 20 to 30 rod photoreceptors. Microvilli extend from the apical surfaces of the RPE cells and interdigitate with photoreceptor outer segments.

Breaking the Cycle. Figure 8 sets out the biochemical reactions involved in retinoid processing and is an attempt to indicate schematically the cellular location of events. In previous work, these reactions have usually been treated as a cycle, but in understanding postbleach recovery, we find it important instead to "break the cycle," by separating (1) those reactions involved in the synthesis and delivery of 11-cis retinoid to the outer segment (solid arrows) from (2) those reactions involved in the removal, processing, and storage of all-trans retinoid after light absorption. Our main reason for emphasizing this distinction stems from our interpretation (discussed later) that, in the normal human eye, the regeneration of rhodopsin after a flash bleach of any magnitude does not require the recycling of retinoid released by the bleach. Thus, it appears that there is a sufficient store of all-trans retinyl ester in the human eye to effectively isolate the synthesis and delivery of 11-cis retinal from the removal and processing of all-trans retinal, at least for a single bleach of up to $100 \%$.

Synthesis and Delivery of 11-cis Retinoid. The human $\mathrm{RPE} /$ choroid contains a large stored quantity of all-trans retinyl ester, amounting, in moles, to approximately 2.5 times the quantity of retinoid bound in rhodopsin. ${ }^{50}$ Delivery of stored ester appears to be mediated by sRPE65, the unpalmitoylated, soluble form of the RPE65 protein, which carries it to the smooth endoplasmic reticulum (SER), where an isomerohydrolase enzyme converts the all-trans ester to the 11-cis alcohol (denoted cis ROL in Fig. 8). Isolation of this enzyme was elusive for many years, but recent evidence has identified it as the palmitoylated, membrane-bound protein mRPE65, possibly in association with other protein(s). ${ }^{51-53}$ The product of this reaction, the 11-cis alcohol, is oxidized by a stereospecific enzyme, 11-cis retinol dehydrogenase (RDH5), to the 11-cis aldehyde (denoted cis RAL). From there, the hydrophobic retinoid is chaperoned to the RPE plasma membrane by cellular retinaldehyde binding protein (CRALBP), and through the interphotoreceptor matrix (IPM) by inter-photoreceptor binding protein (IRBP), eventually finding its way to opsin, with which it recombines to regenerate rhodopsin.

\section{Cellular Model of Recovery Kinetics}

To predict the kinetics of rhodopsin regeneration, it is helpful to redraw the reactions for synthesis and delivery of 11-cis retinoid in a linear arrangement and to show a corresponding hydraulic model, as presented in Figure 9. Here, the black arrows indicate chemical reactions, and the open arrow represents movement down a concentration gradient. In the hy- 


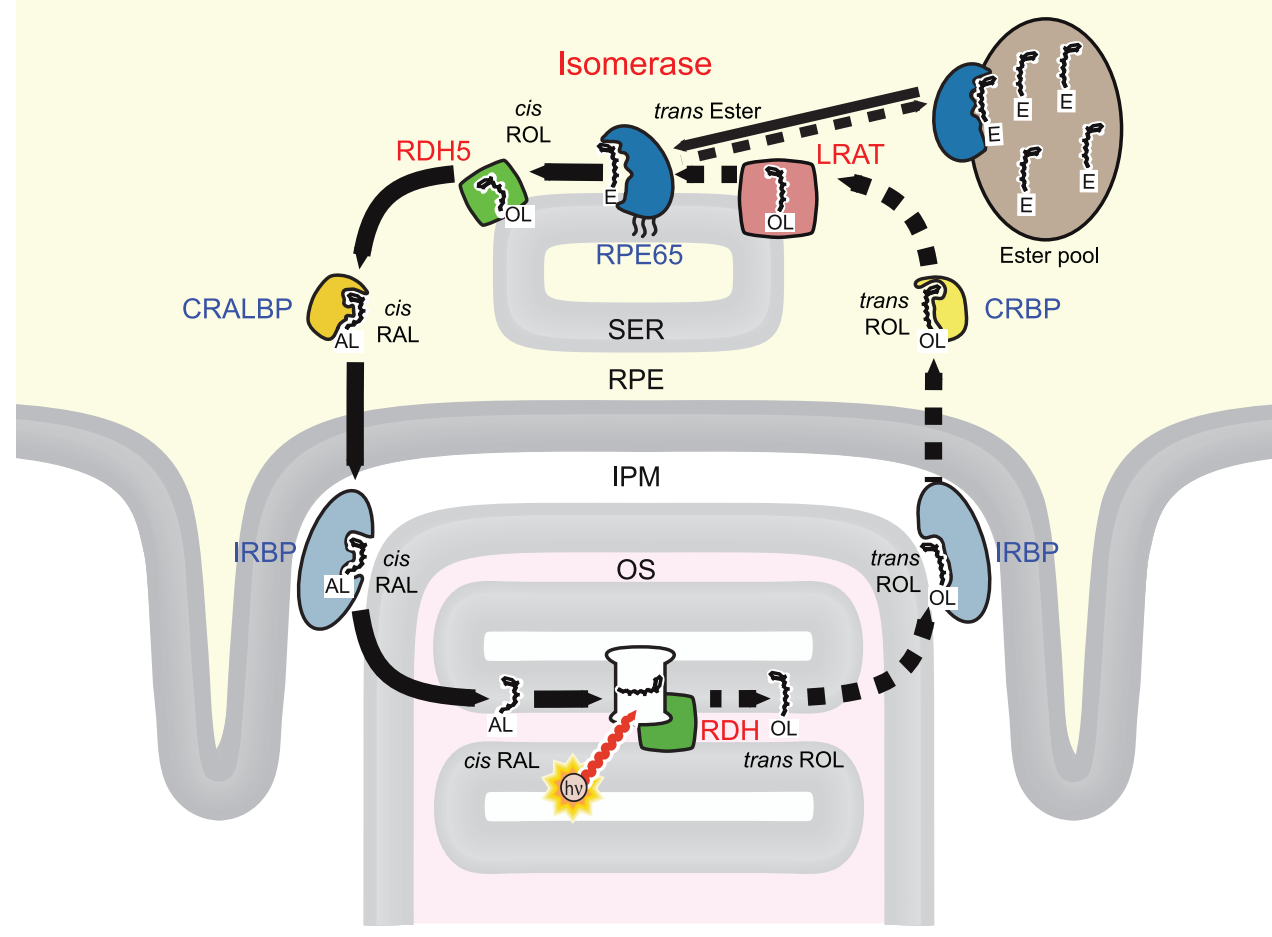

Figure 8. "Breaking the cycle" of biochemical reactions involved in retinoid processing. We have separated the synthesis and delivery of 11-cis retinoid from the removal, processing, and storage of all-trans retinoid by the use of solid and dashed arrows, respectively. OS, outer segment; IPM, interphotoreceptor matrix; RPE, retinal pigment epithelium; SER, smooth endoplasmic reticulum; RAL, retinaldehyde; ROL, retinol. For the chemical icons: $\mathrm{AL}, \mathrm{OL}$, and $\mathrm{E}$ denote the aldehyde, alcohol, and ester groups attached to the retinoid hydrocarbon chain. Enzymes (red): $\mathrm{RDH}$, all-trans retinol dehydrogenase; LRAT, lecithin retinyl acyl transferase; RDH5, 11-cis retinol dehydrogenase. Chaperone proteins (blue): IRBP, interphotoreceptor retinoid binding protein; CRBP, cellular retinol binding protein; RPE65, retinal pigment epithelium $65 \mathrm{kDa}$ protein (which has recently been identified as the isomerase $\left.{ }^{5-53}\right)$; CRALBP, cellular retinaldehyde binding protein.

draulic analogy, the height of each fluid column represents the concentration of the relevant substance (in appropriate units).

In the resting dark steady state, when no retinoid is being delivered to opsin (and on the assumption that there is no loss from the system), then there are no fluxes of retinoid, and hence all the retinoid species are in equilibrium with each other. In terms of the hydraulic analogy, the plug (purple) would be inserted, and the heights of all the fluid columns would be equal (horizontal red lines). But after bleaching, when opsin is formed and is able to bind 11-cis retinal, there is a flow of retinoid and the concentrations are perturbed from the previous equilibrium levels. In the hydraulic model, the plug is removed, and so that fluid flows (blue arrow) as a consequence of pressure differentials between the com- partments. The analysis below is aimed at determining the rate of retinoid flow, as a function of the amount of opsin present.

Resistive Barrier to Diffusion. One scenario that could limit the delivery of 11-cis RAL to opsin is indicated schematically in Figure 9 by the narrowed pipe labeled "Resistance", between the RPE and the rod outer segment. In the presence of a large quantity of opsin (i.e., after extensive bleaching), the concentration of 11-cis retinal in the outer segment drops to a low level, because opsin acts as a sink for this retinoid. If there were a resistance to the delivery of 11-cis retinal from the RPE, then the flow of retinoid would be proportional to the difference in concentration across the barrier, with the flux given by $(C-c) / R$, where $C$ and $c$ denote the concentrations of 11-cis

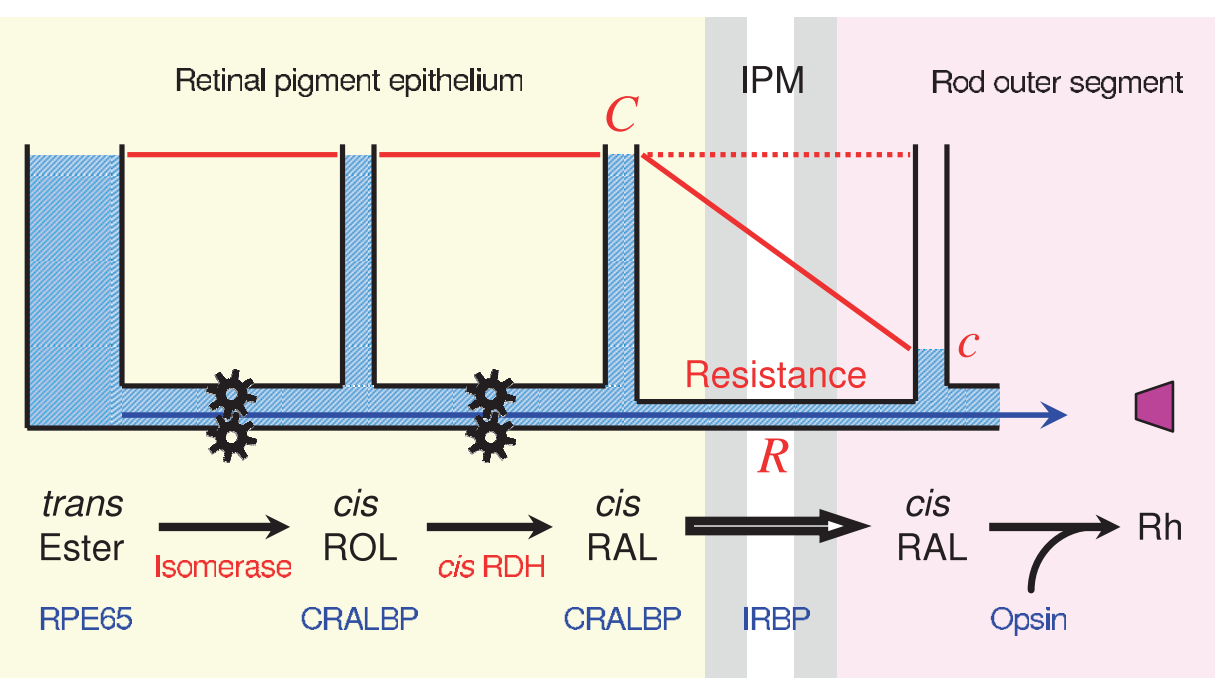

FIGURE 9. Hydraulic analogy of rate limitation in retinoid delivery. The height of each fluid column represents the "concentration" of the retinoid species indicated directly below, in the relevant compartment (RPE or outer segment), and the cogs signify enzymatic conversion steps. In the fully dark-adapted state, when no opsin is present, there is no flux of retinoid out of the system (i.e., the exit pipe is plugged), and the heights of all the fluid columns will be equal. (This finding implies that the heights have been scaled according to the equilibrium constants of the reactions interconverting the substances.) When opsin is produced after bleaching, the local concentration $c$ of 11-cis retinal in the outer segment drops, and retinoid (fluid) flows as a result of the pressure difference, $C-c$. In the case illustrated, the factor limiting the flow is assumed to be the resistance $R$ to delivery of 11-cis retinal to opsin in the outer segments. But, as explained in the text, it is alternatively possible for the limitation to occur at either of the two preceding enzymatic steps: that is, in the hydraulic analogy, if one of the first two horizontal pipes was very narrow. Each of these scenarios generates closely similar rate-limited kinetics of retinoid delivery. 

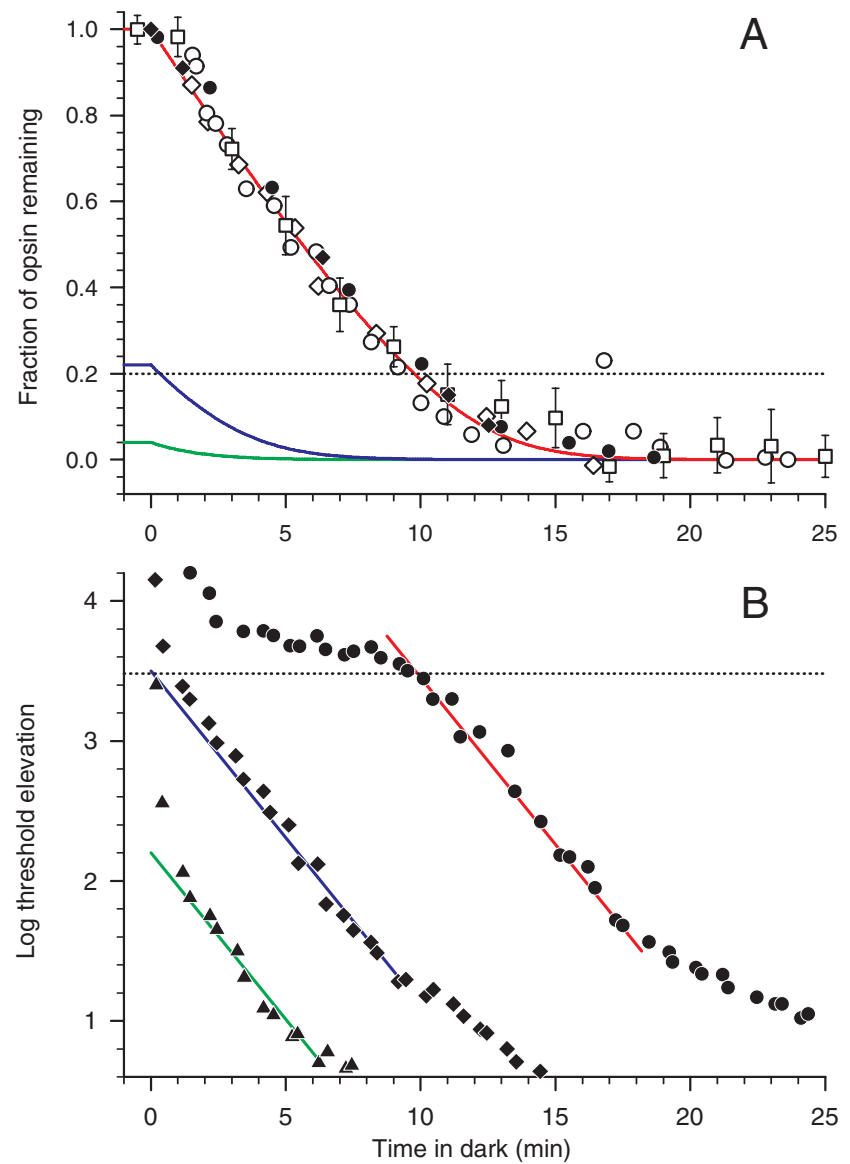

FIGURE 10. Comparison of measured and predicted opsin "removal" by regeneration, and psychophysical dark adaptation, for a normal subject. (A) Declining levels of opsin. Data points correspond to a full bleach, and were obtained by subtracting the rhodopsin regeneration measurements in Figure 7 from unity. Curves are predictions of the rate-limited model, equation 5, for bleaches of $4 \%, 22 \%$ and $100 \%$. Horizontal dotted line represents $20 \%$ opsin remaining. (B) Psychophysical dark adaptation recovery. Data are taken from Figure 6 , for bleaches of $4 \%, 22 \%$, and $98 \%$. Solid lines have a slope of $0.24 \log _{10}$ $\min ^{-1}$, and correspond to the final tail of decline in the respective opsin levels. Dotted borizontal line corresponds to that in (A); thus, when 0.2 of the opsin remained to be "removed", the observer's threshold was elevated by $\sim 3.5 \log$ units.

retinal in the RPE and in the vicinity of opsin, respectively, and where $R$ is the resistance to flow.

This scenario has been analyzed mathematically ${ }^{7,48}$ and has been shown to yield "rate-limited" kinetics of rhodopsin regeneration (discussed later). Thus, the distinct early and late phases of regeneration after a full bleaching (as shown in Figs. 7 and 10) are readily explained on this model. At early times, the high level of opsin causes $c$ to drop to a very low value; but as $c$ cannot drop below zero $(c \geq 0)$, there is a limiting rate of delivery (given by $C / R$ ), corresponding to the initial nearly constant slope in Figures 7 and 10A. Then at a time late in recovery, when regeneration is almost complete and the level of opsin is very small, there is very little retinoid flux and hence very little drop in concentration, so that $c \approx C$. As a result of this nearly fixed concentration, the decay of opsin concentration obeys first-order kinetics, and final recovery follows an exponential time course.

A rate-limited model can account for both the kinetics of rhodopsin regeneration and the kinetics of recovery of scotopic visual threshold, as illustrated in Figure 10. Figure 10A plots the decay of opsin, obtained by inverting the data for rhodopsin from Figure 7 (on the simplifying assumption that the levels of metarhodopsin intermediates can be ignored). The predicted opsin level for a full bleach is plotted by the red curve, and begins declining approximately linearly with time. The final stage of recovery (opsin level below 20\%) is approximately exponential and transforms to a straight line in the semilogarithmic coordinates of the lower panel.

Mathematical Analysis of the Resistive Barrier. In mathematical terms, the time course of "removal" of opsin in the resistive barrier model can be expressed as

$$
\operatorname{Ops}(t)=K_{\mathrm{m}} \mathrm{W}\left\{\frac{B}{K_{\mathrm{m}}} \exp \left(\frac{B}{K_{\mathrm{m}}}\right) \exp \left(-\frac{1+K_{\mathrm{m}}}{K_{\mathrm{m}}} \nu t\right)\right\}
$$

where $O p s(t)$ denotes the proportion of opsin remaining unregenerated at time $t$. The parameters in this equation are: $B$, the fractional bleach; $\nu$, the initial rate of regeneration after a total bleach; and $K_{\mathrm{m}}$, the semisaturation constant (comparable to a $\left.K_{\mathrm{m}}\right)$. The function $\mathrm{W}(x)$ is called the Lambert $\mathrm{W}$ function. ${ }^{54}$ For a complete explanation of this equation, see References 7 and 48.

At times late in recovery, equation 5 predicts that the opsin concentration should decline exponentially, with a time constant of $K_{\mathrm{m}} /\left(1+K_{\mathrm{m}}\right) \nu$. Accordingly, it predicts that the slope of the $\mathrm{S} 2$ region of psychophysical recovery should be given by

$$
\Psi_{\mathrm{S} 2}=\frac{n_{\mathrm{w}}\left(1+K_{\mathrm{m}}\right)}{2.303 K_{\mathrm{m}}} \nu,
$$

where $n_{\mathrm{W}}$ is the exponent of the Weber-Law relation between threshold elevation and background intensity (typically close to unity for large-area scotopic stimuli). Substituting $\nu=0.094$ $\min ^{-1}, K_{\mathrm{m}}=0.17$, and $n_{\mathrm{w}}=0.9$, equation 6 predicts that the S2 slope for a normal subject should be $0.25 \log _{10} \mathrm{~min}^{-1}$, close to the slope observed experimentally. Thus, the model accounts for the experimental measurements quantitatively.

Enzymatic Limit to Retinoid Delivery. An alternative scenario that could limit the rate of rhodopsin regeneration involves the enzymatic processes underlying the synthesis of 11-cis retinal. In terms of the hydraulic analogy in Figure 9, this can be represented by the narrow pipe being placed at (for example) the location of the 11-cis RDH (RDH5), in which case the dominant drop in heights of the fluid columns would occur from 11-cis retinol (cis ROL) to 11-cis retinal (cis RAL). Alternatively, the limit could occur at the isomerase. Mathematical analysis of Michaelis-Menten enzyme kinetics (Appendix) shows that in such cases fundamentally the same kind of rate-limiting behavior of retinoid delivery is obtained as for the resistive barrier considered previously. Hence, it is possible for an enzymatic limit to explain the observed kinetics.

However, in the normal human eye, we think it unlikely that the limiting step is an enzymatic one, though we cannot entirely rule out this possibility. The kind of evidence that would be needed to support an enzymatic limit would be a finding of substantially slowed postbleach recovery in human subjects carrying a single normal allele of RDH5 or of RPE65. Thus, one would expect that if either the 11-cis retinol dehydrogenase or the isomerase were rate-limiting for regeneration in normal subjects, then halving that enzyme's expression level would halve the initial rate $(\nu)$ of regeneration. In the case of the RPE65, it has been reported that three heterozygote individuals displayed normal dark adaptation, ${ }^{55}$ whereas in the case of RDH5 we are not aware of dark adaptation measurements on heterozygotes. For both these enzyme, it would be very illuminating to investigate further the kinetics of dark adaptation in individuals carrying a single functional copy of the gene. 


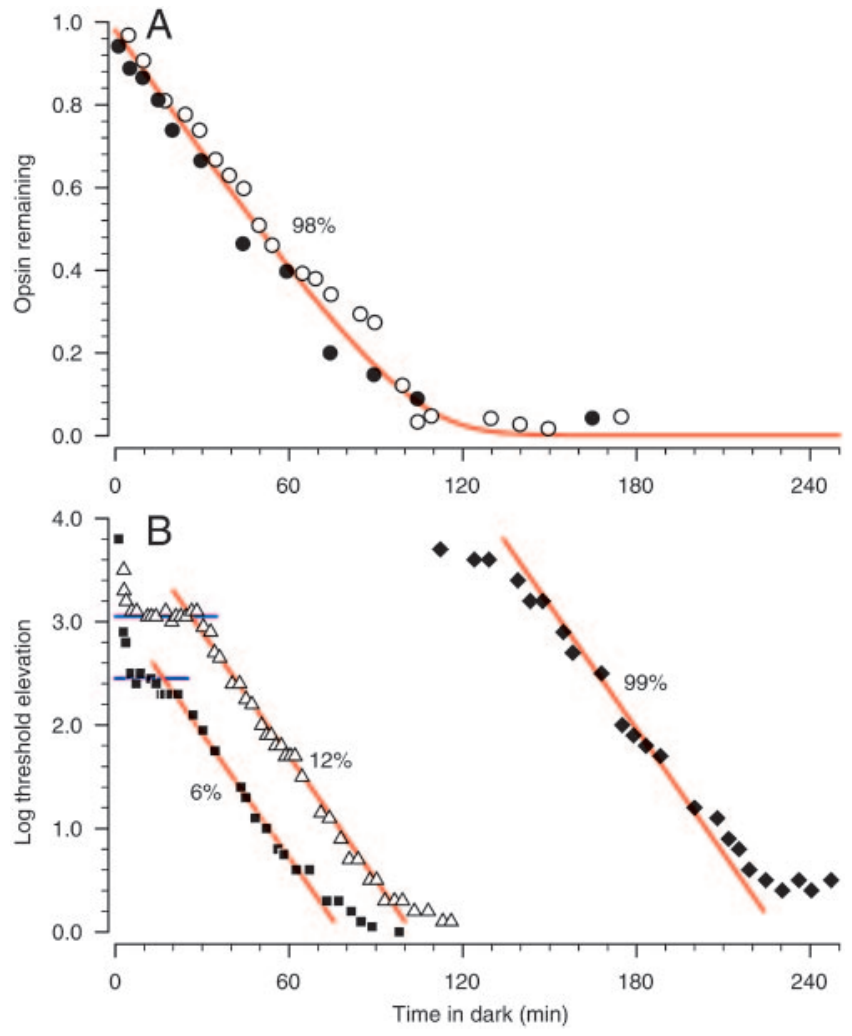

FIGURE 11. Fundus albipunctatus: Measured and predicted rhodopsin regeneration and psychophysical dark adaptation, from studies separated by more than two decades. (A) Recovery of rhodopsin, measured by reflection densitometry after a large bleach, for two brothers with fundus albipunctatus $(\bullet),(\bigcirc)$; data from Reference 56 . Curve plots the rate-limited prediction of equation 4, with $\nu=0.01 \mathrm{~min}^{-1}, K_{\mathrm{m}}=0.1$, and $B=0.98$ (98\% bleach). (B) Time course of dark adaptation in a different patient, after bleach of three magnitudes: $(\square) 6 \%$; $\triangle$ ) $12 \%$; (๑) 99\%; data from Reference 57. Red lines have a common S2 slope of $0.04 \log _{10}$ unit $\mathrm{min}^{-1}$; borizontal blue lines in (B) indicate rod plateaux for the smaller bleaches (see text). Replotted from Figure 18 of Reference 7.

Interpretations. In summary, our interpretations of postbleaching recovery kinetics are as follows. First, we think that in the normal human eye, the rate-limited regeneration of rhodopsin and the S2 phase of dark adaptation have a common origin in the existence of a resistive barrier between a source of 11-cis retinal (in the RPE) and the opsin molecules in the outer segments. We are not sure what constitutes this barrier, but we suggest that it may occur substantially within the outer segment (e.g., from the plasma membrane to the disc membrane). Second, we think that in certain disease states, the rate of regeneration could drop to a substantially lower level as a result of reduced enzyme activity, while still being described by a broadly similar rate-limited initial slope and final S2 component. An example of such an enzymatic limit occurs in fundus albipunctatus, as we now describe.

\section{Diseases Exhibiting Slowed Dark Adaptation}

Fundus Albipunctatus (11-cis Retinol Dehydrogenase, RDH5). Measurements of rhodopsin regeneration and dark adaptation are illustrated in Figure 11, from two studies in the literature separated by more than 25 years. ${ }^{56,57}$ Figure $11 \mathrm{~A}$ shows that regeneration is again rate limited in this disease, though at a speed of approximately one ninth of normal, whereas Fig. 11B shows that dark adaptation again exhibits an S2 region, though with a slope approximately one sixth of
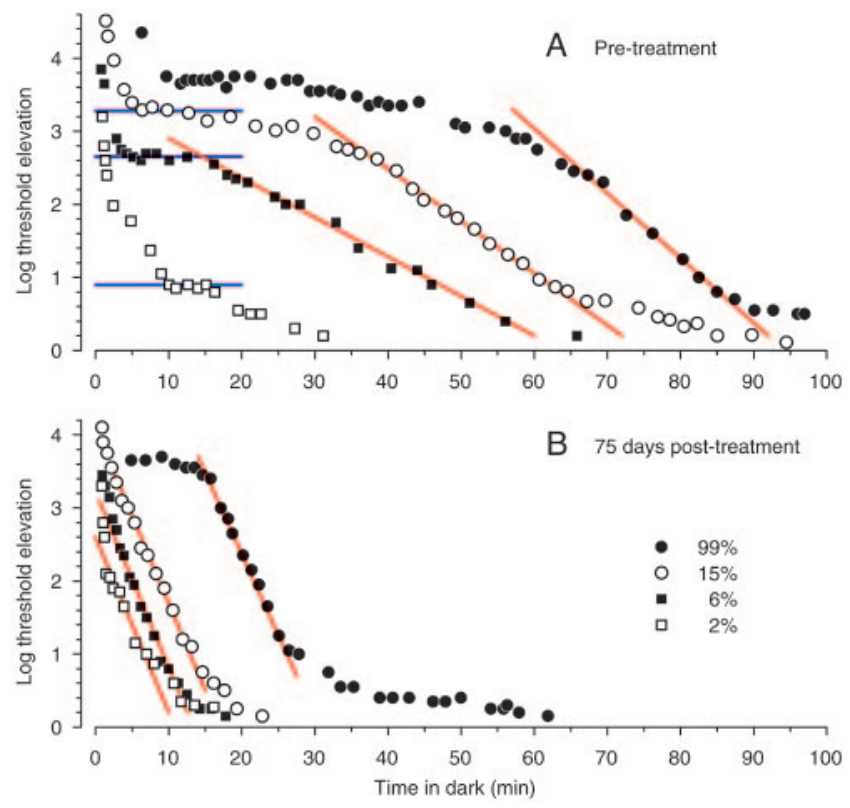

FigURE 12. Systemic VAD: psychophysical dark adaptation. (A) Measurements of dark adaptation on presentation of the patient. (B) After 75 days of supplementation with vitamin A. Data from Figure 2 of Reference 58. The key for bleach levels in (B) also applies in (A). Red lines: component S2; the slopes were: 0.054, 0.071, and $0.089 \log _{10}$ $\min ^{-1}$ in (A); and $0.24 \log _{10} \mathrm{~min}^{-1}$ in (B). (A, borizontal blue lines) Rod plateaux for the smaller bleaches (see text). Replotted from Figure 17 of Reference 7 .

normal (in a different patient). Of importance, the final rhodopsin levels and the final visual thresholds are entirely normal, indicating that the retina is otherwise functioning normally.

The lowered rate of rhodopsin regeneration presumably reflects the lowered rate of alcohol oxidation that can be provided by nonspecific dehydrogenases, which are presumed to generate 11-cis retinal from 11-cis retinol in the absence of the normal dehydrogenase enzyme (RDH5). We interpret the lowered S2 slope to indicate a lowered steady state concentration $C$ of 11-cis retinal in the RPE. ${ }^{7}$ To account for this lowered steady state concentration, we propose that there must be

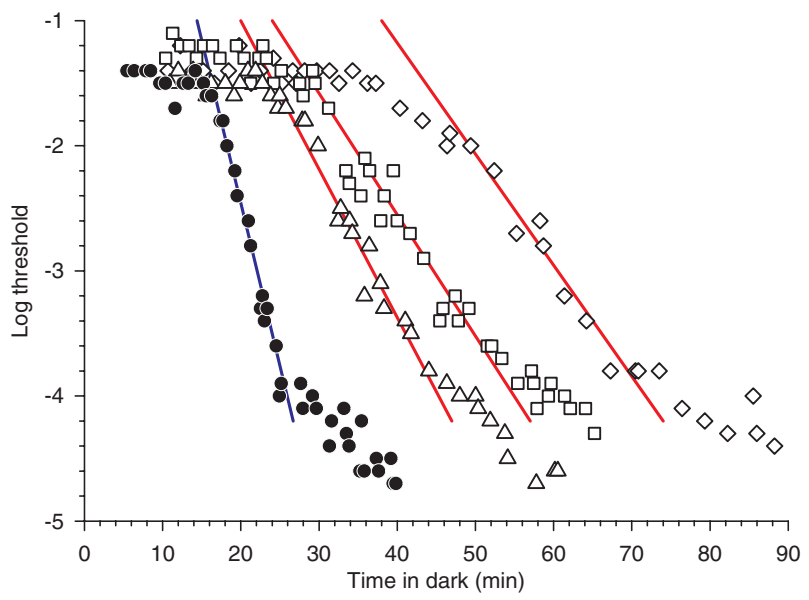

FIGURE 13. Age-related maculopathy: psychophysical dark adaptation. Recovery of $\log$ threshold for three patients with early ARM (open symbols), and for one healthy subject of similar age (filled symbol), after bleaches of 98\%. Data from Figure 2 of Reference 61. Straight lines: component S2 of recovery, with slopes of $0.26(\bullet), 0.12(\triangle)$, $0.097(\square)$, and $0.089(\diamond) \log _{10} \mathrm{~min}^{-1}$. 
some kind of "leakage" pathway, so that, even in the absence of opsin, the 11-cis retinoid is being used up; hence, with lowered synthesis there would be a lowered concentration of 11-cis retinal.

Systemic Vitamin A Deficiency. Another disease that is readily understood in terms of the hydraulic analogy is systemic vitamin A deficiency (VAD). A deficiency in retinoid levels would be expected to lower the stored amount of alltrans retinyl ester, and hence, in terms of Figure 9, to lower the height of fluid in the lefthand reservoir; in this case the predicted flow rate would simply be scaled down from normal due to the lowered pressure.

Dark adaptation measurements from a VAD patient are illustrated in Figure $12^{58}$, both on presentation (Fig. 12A) and after 10 weeks of vitamin A supplementation (Fig. 12B). As for the patient with fundus albipunctatus in Figure 11B, the final thresholds were entirely normal. Furthermore, the kinetics of recovery returned substantially toward normal within 24 hours of vitamin A supplementation ${ }^{58}$ (not shown). These two observations strongly support the notion that no pathology was present apart from retinoid deficiency.

In the deficient state, recovery after each bleach exhibited an approximately straight-line region (red lines), which we interpret as component S2; the magnitude of this slope was considerably reduced from that in Figure 6, being no more than $40 \%$ of normal. For the $6 \%$ bleach, the S2 slope was $0.054 \log _{10}$ $\min ^{-1}$, less than one fourth of normal, and we suggest that this value provides a measure of the relative concentration of 11-cis retinal in the RPE of this patient under fully dark-adapted conditions. For the two larger bleaches (15\% and 99\%), the slope of the S2 component was somewhat higher, and we attribute this to transient refilling of the ester pool as a result of recycling of released all-trans retinoid. This is the only example, of which we are aware, of an observable effect of retinoid recycling on human dark adaptation after a single bleach.

A noteworthy feature at early times after small bleaches is the occurrence of a plateau region (blue lines), during which it has clearly been established that detection is mediated by the rods rather than the cones. ${ }^{58}$ Similar plateau behavior may be seen for the fundus albipunctatus patient after the smaller bleaches in Figure 11B. We attribute these rod plateaux to transient exhaustion the retinyl ester store.

Finally, we note that in this VAD patient the S2 slope returned to the normal value of $0.24 \log _{10} \mathrm{~min}^{-1}$ after 10 weeks of supplementation with vitamin A (Fig. 12B).

Age-Related Maculopathy. Dark adaptation is slowed in age-related maculopathy (ARM). ${ }^{59-61}$ Measurements from three elderly patients with early ARM are presented in Figure 13 and are compared with measurements from a healthy subject of similar age. ${ }^{61}$ It is clear that recovery occurs more slowly in these patients, and it is notable that the slope of the S2 region (red lines) is substantially lower than that in the healthy subject (blue line).

In the 20 patients with early ARM (mean age, 75 years) examined in that study, the S2 slope had a mean of $0.15 \log _{10}$ $\min ^{-1}$, compared with a mean of $0.23 \log _{10} \min ^{-1}$ for 16 subjects of similar age (mean, 72 years) with fundi of normal appearance. ${ }^{61}$ Thus the 52 slope was approximately two thirds of normal in patients with early ARM. In addition, in those patients, the time to the cone-rod break was substantially delayed.

Characteristic changes in ARM include a thickening of Bruch's membrane and the deposition of neutral lipids, so that the region becomes hydrophobic (e.g., see Refs. 62-65). Hence, the likely explanation for the slowing of dark adaptation in ARM is that the transport of vitamin A from the choroidal circulation is hindered by these changes, ${ }^{61,66}$ so that the
RPE/retina becomes vitamin A deficient-a situation that we refer to as ocular VAD.

It has been proposed that the reduced level of vitamin $\mathrm{A}$ is causal for the subsequent retinal degeneration in AMD. ${ }^{66}$ This seems unlikely to us, and we think it much more likely that degeneration results from other consequences of the "clogging-up" of Bruch's membrane. A wealth of recent genetic and histopathological information has pointed to an aberrant inflammatory response in the neighborhood of Bruch's membrane as a major causal factor in the development of advanced ARM (reviewed in Ref. 67). The transport of many metabolites into, and waste products out of, the RPE/retina is very likely to be disrupted in ARM, and it could be that the levels of a combination of these substances are crucial to photoreceptor survival. The Age-Related Eye Disease Study ${ }^{68}$ reported that supplementation with a high dose of antioxidants $(500 \mathrm{mg}$ vitamin $C$ and $400 \mathrm{IU}$ vitamin E) in conjunction with $15 \mathrm{mg}$ $\beta$-carotene led to a reduction of approximately $20 \%$ in the probability of progression to advanced forms of AMD (an odds ratio of 0.8 ) over a 6-year study period. Of importance, no statistically significant serious adverse effects were reported. Nevertheless, we suggest that further study may be warranted, given that retinoids form a component of the lipid deposits in Bruch's membrane.

Measurement of the slowing of dark adaptation is likely to provide a valuable and noninvasive bioassay, both as a diagnostic tool for predicting the likelihood of onset of macular degeneration and as a means of assessing the efficacy of treatments of the disease.

Other Diseases Affecting Dark Adaptation. Several other diseases affect dark adaptation, and we very briefly consider them now (for further details, see Refs. 7,69-71).

Oguchi Disease. Oguchi disease is caused by a mutation in one or other of two proteins involved in shut-off of the phototransduction cascade: rhodopsin kinase or arrestin. Hence, this disease does not involve retinoid chemistry, and accordingly its features are very different from those of most other diseases exhibiting slowed dark adaptation. In particular, the kinetics of regeneration of rhodopsin appear essentially normal, despite drastically slowed psychophysical dark adaptation. The slowed dark adaptation appears to be explicable in terms of the presence of nonquenched $\mathrm{R}^{*}$ (activated rhodopsin), rather than of opsin. . $^{7,72}$

Sorsby Fundus Dystrophy. Sorsby fundus dystrophy is caused by a mutation in TIMP3, a metalloproteinase of the matrix in Bruch's membrane. All the features of this disease are consistent with the slowing of dark adaptation being caused by ocular VAD. ${ }^{58,73}$

RPE65. RPE65, acting alone or perhaps in concert with one or more additional proteins, is thought to be the isomerase, the enzyme that converts all-trans retinyl ester into 11-cis retinol (Fig. 8). Mutations in RPE65 give rise to autosomal recessive, severe retinal dystrophy, with childhood onset (reviewed in Ref. 70). Although heterozygotes with one normal allele exhibit mild ophthalmoscopic abnormalities, they have been reported to dark adapt normally, ${ }^{55}$ implying that RPE65 is not rate-limiting in dark adaptation in the normal human eye.

Bothnia Dystrophy. Several rare diseases, of which Bothnia dystrophy is an example, involve mutations in CRALBP, the chaperone protein for 11-cis retinal (and possibly for 11-cis retinol). In these patients, loss of CRALBP leads to enormously slowed dark adaptation, ${ }^{74}$ in which the $\$ 2$ slope is reduced by $\sim 20$-fold. Correspondingly, in knockout mice $\left(R l b p 1^{-/}\right)$, the loss of CRALBP leads to at least a 10-fold slowing of both rhodopsin regeneration and recovery of rod circulating cur- 
rent. ${ }^{75}$ Thus, it appears that the effective concentration of 11-cis retinal is lowered by the absence of chaperone.

Stargardt Macular Dystrophy. Stargardt macular dystrophy is caused by a mutation in the ABCR protein that is thought to extrude the condensation product of 11-cis retinal bound to lipid from the interior of the disc membrane. ${ }^{76,77}$ The devastating symptoms of the disease are thought to be caused by the presence of lipofuscin, and in particular its major component A2E, a retinoid byproduct. ${ }^{78}$ Measurements of dark adaptation $^{79,80}$ show that the slope of the S2 phase of dark adaptation is unaltered. This indicates that, irrespective of whether some appreciable fraction of bleach-released all-trans retinal exits via the disc interior and extrusion by the ABCR transporter, the time course of dark adaptation is not critically dependent on such fluxes.

\section{SUMMARY}

\section{Phototransduction}

The activation steps of the phototransduction cascade are understood in considerable detail at a molecular level. We developed a mathematical model of these steps that provides an accurate description of the onset phase of the light response, as measured from individual rods or from the scotopic ERG and that allows measurement of a fundamental parameter of the cascade: its amplification. The overall amplification of the electrical response is explicable as the product of gain factors arising in the underlying physical and biochemical steps, and it is straightforward to account for the differences in amplification between amphibian and mammalian rods.

\section{Dark Adaptation}

Our results and analysis support the hypothesis that during postbleach dark adaptation recovery, the elevated visual threshold in the "S2" phase is caused by the presence of opsin, which weakly activates the transduction cascade. The slowness of recovery results from rate-limited delivery of 11-cis retinal to recombine with opsin. The cause of the rate limitation can be either a resistive barrier to delivery (which we think applies to recovery in the normal human eye), or else an enzymatic limitation in the processing of 11-cis retinoid (which applies in some diseases). Measurement of the S2 slope in human subjects may provide an important bioassay for certain diseases, notably in the case of age-related maculopathy.

\section{APPENDIX}

\section{Derivation of Rate-Limited Kinetics for Enzymatic Production of Retinoid}

In this section, we derive equations showing that a MichaelisMenten enzyme reaction for production of 11-cis retinoid can generate rate-limited kinetics of rhodopsin regeneration, very similar to the kinetics generated by the resistive barrier model considered previously. ${ }^{7,48}$

Consider the conversion of a substrate $S$ into a product $P$, by an enzyme $\mathrm{E}$, with the substrate and product binding to the enzyme to form an enzyme-intermediate complex, E·I, according to the reversible reaction scheme

$$
\mathrm{S}+\mathrm{E} \underset{\boldsymbol{k}_{\mathrm{IS}}}{\stackrel{\boldsymbol{k}_{\mathrm{SI}}}{\leftrightarrows}} \mathrm{E} \cdot \underset{\mathrm{I}}{\stackrel{\boldsymbol{k}_{\mathrm{IP}}}{\Longleftrightarrow}} \mathrm{E}+\mathrm{P} .
$$

If the concentration of the product $P$ is varied, in the face of a constant concentration of substrate $S$, then the following occurs. At low concentrations of $\mathrm{P}$, the reaction proceeds in the forward direction; at an equilibrium concentration of $P$, the net reaction rate is zero; and at even higher concentrations of $\mathrm{P}$, the reaction proceeds in the reverse direction. The following analysis represents a case of the more general King-Altman method (e.g., see Ref. 81).

The rate $r$ of the forward reaction (conversion of substrate into product) can be written as

$$
r=k_{\mathrm{SI}} S E-k_{\mathrm{IS}} I=k_{\mathrm{IP}} I-k_{\mathrm{PI}} P E
$$

where $S$ and $P$ denote the concentrations of the species $S$ and $\mathrm{P}$, and where $E$ and $I$ denote the concentrations of free enzyme $\mathrm{E}$ and of enzyme bound to intermediate, E$\cdot \mathrm{I}$, respectively. Conservation requires the total quantity of enzyme to be constant, at $E_{\text {tot }}=E+I$.

The concentration $E$ of free enzyme can be obtained by solving the two simultaneous equations in A2, along with the conservation relation, to yield

$$
E=\frac{E_{\text {tot }}}{1+S / K_{\mathrm{S}}+P / K_{\mathrm{P}}},
$$

where $K_{\mathrm{S}}$ and $K_{\mathrm{P}}$ are the Michaelis constants for S and $\mathrm{P}$, given by $K_{\mathrm{S}}=\left(k_{\mathrm{IS}}+k_{\mathrm{IP}}\right) / k_{\mathrm{SI}}$ and $K_{\mathrm{P}}=\left(k_{\mathrm{IS}}+k_{\mathrm{IP}}\right) / k_{\mathrm{PI}}$.

Substituting equation $\mathrm{A} 3$ into $\mathrm{A} 2$, the rate of reaction can be written as

$$
r=E\left(k_{\mathrm{IP}} \frac{S}{K_{\mathrm{S}}}-k_{\mathrm{IS}} \frac{P}{K_{\mathrm{P}}}\right)
$$

where the sign of the term in parentheses determines whether the reaction proceeds in the forward or reverse direction.

We now specify that the concentration of substrate is held constant at $S=S_{0}$, and we denote the corresponding equilibrium concentration of the product as $P_{0}$; this is the concentration of product that elicits zero net flux for the reaction. By setting $r=0$ in equation A4, the equilibrium concentration $P_{0}$ is seen to satisfy

$$
\frac{P_{0}}{S_{0}}=\frac{k_{\mathrm{IP}} K_{\mathrm{P}}}{k_{\mathrm{IS}} K_{\mathrm{S}}}=K
$$

where $K$ is defined as the equilibrium constant for the conversion of $\mathrm{S}$ into $\mathrm{P}$.

Substituting equation A5 into A4, and setting $S=S_{0}$, we obtain the forward reaction rate $r$ as

$$
r=k_{\mathrm{IP}} E_{\mathrm{tot}} \frac{S_{0}}{K_{\mathrm{S}}}\left(1-\frac{P}{P_{0}}\right) /\left(1+\frac{S_{0}}{K_{\mathrm{S}}}+\frac{P_{0}}{K_{\mathrm{P}}} \frac{P}{P_{0}}\right) .
$$

Here, the term $\left(1-P / P_{\mathrm{O}}\right)$ provides the required reversibility behavior. Thus, the reaction is in equilibrium when $P=P_{\mathrm{O}}$, and proceeds in the forward direction when $P<P_{0}$, and in the reverse direction when $P>P_{0}$. Furthermore, substitution of $P=0$ yields the maximum forward rate as $r_{\max }=k_{\mathrm{IP}}\left\{E_{\mathrm{tot}} S_{\mathrm{o}}\right\}$ $\left.\left(S_{\mathrm{O}}+K_{\mathrm{S}}\right)\right\}$. This result is as expected, because the term in braces specifies the amount of $S$ bound to enzyme (as species $\mathrm{E} \cdot \mathrm{I})$ in the absence of $\mathrm{P}$.

\section{Implications for the Rate of Rhodopsin Regeneration}

Previously, ${ }^{7,48}$ we have considered the case in which the enzymatic reactions cause no limit (i.e., where the concentration $C$ of 11-cis retinal in the RPE is constant) and where the limitation is caused solely by a resistive barrier to diffusion. 
Now we consider the converse case, when there is no resistive barrier (so $c=C$ ), and instead an enzymatic reaction is limiting.

First, we consider the case in which the enzyme E represents the 11-cis RDH (RDH5) (i.e., when the product $\mathrm{P}$ is 11-cis retinal). Because we have now eliminated any barrier between the RPE and opsin, $P$ represents the concentration $c$ of 11-cis retinal in the vicinity of opsin. As set out in Section 4.2.1 of Reference 7 , the rate of binding of 11-cis retinal to opsin is proportional to the product of $c$ and the amount of opsin remaining available. Hence, the situation is closely similar to that for the resistive barrier model. After a very large bleach, the concentration of 11-cis retinal will drop to a low level, $P \rightarrow 0$, and the enzymatic reaction will operate at a rate approaching its maximum forward rate. But when regeneration is almost complete, and there is little flux, then the concentration of 11-cis retinal will approach a constant level, $P \rightarrow P_{0}$, and the final decay of opsin will again follow an exponential time course.

The rate of binding of 11-cis retinal to opsin is described by the bimolecular combination

$$
\mathrm{dOps}(t) / d t=-k P(t) O p s(t)
$$

exactly as in the case of equation 1 in Reference 7 . We have been unable to obtain an analytical solution comparable to that in equation 5 , but numerical simulations show that the form of the predicted recovery is closely similar to the rate-limited shape plotted in Figure 10, for reasonable values of the parameters in equation A6.

In the second case, where the enzyme $\mathrm{E}$ represents the isomerase, the analysis is more complicated. But we think that the solution is likely to exhibit a similar rate-limited shape.

\section{Acknowledgments}

We gratefully acknowledge the contributions of our colleagues Arkady Lyubarsky, Omar Mahroo, Sergei Nikonov, and Gary Whitlock, who participated over a number of years in this work. We are very grateful to the following investigators for providing data in digital form: Marie Burns for the image and data plotted in Figure 5; Jan van de Kraats and Dirk van Norren for unpublished data plotted in Figures 7 and 10; and Cynthia Owsley for data plotted in Figure 11.

\section{References}

1. Lamb TD, Pugh EN Jr. A quantitative account of the activation steps involved in phototransduction in amphibian photoreceptors. J Pbysiol. 1992;449:719-758.

2. Pugh EN Jr, Lamb TD. Amplification and kinetics of the activation steps in phototransduction. Biochim Biopbys Acta. 1993;1141: 111-149.

3. Pugh EN Jr, Lamb TD. Phototransduction in vertebrate rods and cones: molecular mechanisms of amplification, recovery and light adaptation. In: Handbook of Biological Physics, Vol. 3. Amsterdam: Elsevier; 2000:183-255.

4. Arshavsky VY, Lamb TD, Pugh EN Jr. G proteins and phototransduction. Ann Rev Physiol. 2002;64:153-187.

5. Burns ME, Lamb TD. Visual transduction by rod and cone photoreceptors. In: Chalupa LM, Werner JS, ed. The Visual Neurosciences. Cambridge, MA: MIT Press;1993:215-233.

6. Fisher SK, Anderson DH, Erickson PA, Guérin CJ, Lewis GP, Linberg KA. Light and electron microscopy of vertebrate photoreceptors. In: Hargrave PA, ed. Methods in Neurosciences. Vol. 15. 1993:3-36.

7. Lamb TD, Pugh EN Jr. Dark adaptation and the retinoid cycle of vision. Prog Retinal Eye Res. 2004;23:307-380.

8. Pugh EN Jr, Nikonov S, Lamb TD. Molecular mechanisms of vertebrate photoreceptor light adaptation. Curr Opin Neurobiol. 1999;9:410 - 418 .
9. Leskov IB, Klenchin VA, Handy JW, et al. The gain of rod phototransduction: reconciliation of biochemical and electrophysiological measurements. Neuron. 2000;27:525-537.

10. Baylor DA, Nunn BJ. Electrical properties of the light-sensitive conductance of rods of the salamander Ambystoma tigrinum. J Pbysiol. 1986;371:115-145.

11. Torre V, Matthews HR, Lamb TD. Role of calcium in regulating the cyclic GMP cascade of phototransduction in retinal rods. Proc Natl Acad Sci USA. 1986;83:7109-7113.

12. Kraft TW, Schneeweis DM, Schnapf JL. Visual transduction in human rod photoreceptors. J Physiol. 1993;464:747-765.

13. Lyubarsky AL, Pugh EN Jr. Recovery phase of the murine rod photoresponse reconstructed from electroretinographic recordings. J Neurosci. 1996;16:563-571.

14. Friedburg C, Thomas MM, Lamb TD. Time course of the flash response of dark- and light-adapted human rod photoreceptors derived from the electroretinogram. J Physiol. 2001;534:217-242.

15. Smith NP, Lamb TD. The a-wave of the human electroretinogram recorded with a minimally invasive technique. Vision Res. 1997; 37:2943-2952.

16. Brown KT, Wiesel TN. Localization of origins of electroretinogram components by intraretinal recording in the intact cat eye. J Physiol. 1961;158:257-280.

17. Hagins WA, Penn RD, Yoshikami S. Dark current and photocurrent in retinal rods. Biophys J. 1970;10:380-412.

18. Kang Derwent JJ, Linsenmeier RA. Intraretinal analysis of the a-wave of the electroretinogram (ERG) in dark-adapted intact cat retina. Vis Neurosci. 2001;18:353-363.

19. Jamison JA. Rod photoreceptor contribution to the mammalian electroretinogram. PhD thesis. Ann Arbor, MI: University of Michigan; 2003.

20. Breton ME, Schueller AW, Lamb TD, Pugh EN Jr. Analysis of ERG a-wave amplification and kinetics in terms of the G-protein cascade of phototransduction. Invest Ophthalmol Vis Sci. 1994;35:295309.

21. Nikonov SS, Daniele LL, Zhu X, Craft CM, Swaroop A, Pugh EN Jr. Photoreceptors of $\mathrm{Nrl}^{-/-}$mice coexpress functional S- and M-cone opsins having distinct inactivation mechanisms. J Gen Physiol. 2005;125:287-304.

22. Nymark S, Heikkinen H, Haldin C, Donner K, Koskelainen A. Light responses and light adaptation in rat retinal rods at different temperatures. J Physiol. 2005;567:923-938.

23. Burns ME, Mendez A, Chen C-K, et al. Deactivation of phosphorylated and nonphosphorylated rhodopsin by arrestin splice variants. J Neurosci. 2006;26:1036-1044.

24. Whitlock GG, Lamb TD. Variability in the time course of single photon responses from toad rods: termination of rhodopsin's activity. Neuron. 1999;23:337-351.

25. Baylor DA, Burns ME. Control of rhodopsin activity in vision. Eye. 1998; 12:521-525.

26. Lamb TD, Matthews HR, Torre V. Incorporation of calcium buffers into salamander retinal rods: a rejection of the calcium hypothesis of phototransduction. J Pbysiol. 1986;372:315-349.

27. Dowling JE. The chemistry of visual adaptation in the rat. Nature. 1960;188:114-118.

28. Rushton WAH. The Ferrier Lecture, 1962. Visual adaptation. Proc Roy Soc B. 1965;162:20-46.

29. Lamb TD. The involvement of rod photoreceptors in dark adaptation. Vision Res. 1981;21:1773-1782.

30. Pugh EN Jr. Rushton's paradox: rod dark adaptation after flash photolysis. J Physiol. 1975;248:413-431.

31. Jackson GR, Owsley C, McGwin G Jr. Aging and dark adaptation. Vision Res. 1999;39:3975-3982.

32. Stiles WS, Crawford BH. Equivalent adaptational levels in localized retinal areas. In: Report of a Joint Discussion on Vision. Cambridge, UK: Cambridge University Press; 1932:194-211.

33. Lamb TD, Cideciyan AV, Jacobson SG, Pugh EN Jr. Towards a molecular description of human dark adaptation. J Physiol. 1998; 506:88P.

34. Cornwall MC, Fain GL. Bleached pigment activates transduction in isolated rods of the salamander retina. J Physiol. 1994;480:261279. 
35. Melia TJ, Cowan CW, Angleson JK, Wensel TG. A comparison of the efficiency of $G$ protein activation by ligand-free and lightactivated forms of rhodopsin. Biophys J. 1997;73:3182-3191.

36. Pepperberg DR, Brown PK, Lurie M, Dowling JE. Visual pigment and photoreceptor sensitivity in the isolated skate retina. $J$ Gen Pbysiol. 1978;71:369-396.

37. Corson DW, Cornwall MC, MacNichol EF, et al. Sensitization of bleached rod photoreceptors by 11-cis-locked analogues of retinal. Proc Natl Acad Sci USA. 1990;87:6823-6827.

38. Hagins WA, Rushton WAH. The measurement of rhodopsin in the decerebrate albino rabbit. J Pbysiol. 1953;120:61P.

39. Rushton WAH, Campbell FW, Hagins WA, Brindley GS. The bleaching and regeneration of rhodopsin in the living eye of the albino rabbit and man. Optica Acta. 1955;1:183-190.

40. van de Kraats J, Berendschot TTJM, van Norren D. The pathways of light measured in fundus reflectometry. Vision Res. 1996;36: 2229-2247.

41. Alpern M. Rhodopsin kinetics in the human eye. J Pbysiol. 1971; 217:447-471.

42. Rushton WAH, Powell DS. The rhodopsin content and the visual threshold of human rods. Vision Res. 1972;12:1073-1081.

43. van Norren D, van de Kraats J. Retinal densitometer with the size of a fundus camera. Vision Res. 1989;29:369-374.

44. Liem AT, Keunen JE, van Norren D, van de Kraats J. Rod densitometry in the aging human eye. Invest Opbthalmol Vis Sci. 1991;32:2676-2682.

45. Thomas MM, Lamb TD. Light adaptation and dark adaptation of human rod photoreceptors measured from the $a$-wave of the electroretinogram. J Physiol. 1999;518:479-496.

46. Bonds AB, MacLeod DIA. The bleaching and regeneration of rhodopsin in the cat. J Pbysiol. 1974;242:237-253.

47. Ripps H, Mehaffey L III, Siegel IM. Rhodopsin kinetics in the cat retina. J Gen Physiol. 1981;77:317-334.

48. Mahroo OAR, Lamb TD. Recovery of the human photopic electroretinogram after bleaching exposures: estimation of pigment regeneration kinetics. J Physiol. 2004;554:417-437.

49. Bok D. The retinal pigment epithelium, a versatile partner in vision. J Cell Sci. 1993;17:189-195.

50. Bridges CD, Alvarez RA, Fong S-L. Vitamin A in human eyes: amount, distribution, and composition. Invest Ophthalmol Vis Sci. 1982;22:706-714.

51. Jin MH, Li SH, Moghrabi WN, Sun H \& Travis GH. Rpe65 is the retinoid isomerase in bovine retinal pigment epithelium. Cell. 2005;122:449-459.

52. Moiseyev G, Chen Y, Takahashi Y, Wu BX, Ma J. RPE65 is the isomerohydrolase in the retinoid visual cycle. Proc Natl Acad Sci USA. 2005;102:12413-12418.

53. Redmond TM, Poliakov E, Yu S, Tsai J-Y, Lu Z, Gentleman, S. Mutation of key residues of RPE65 abolishes its enzymatic role as isomerohydrolase in the visual cycle. Proc Natl Acad Sci USA. 1005; 102:13658-13663.

54. Corless RM, Gonnet GH, Hare DEG, Jeffrey DJ, Knuth DE. On the Lambert W function. Adv Comput Math. 1996;5:329-359.

55. Hamel CP, Griffoin J-M, Lasquellec L, Bazalgette C, Arnaud B. Retinal dystrophies caused by mutations in RPE65: assessment of visual functions. Br J Ophthalmol. 2001;85:424-427.

56. Carr RE, Ripps H, Siegel IM. Visual pigment kinetics and adaptation in fundus albipunctatus. Doc Opbthalmol Proc Series. 1974;4:193204.

57. Cideciyan AV, Haeseleer F, Fariss RN, et al. Rod and cone visual cycle consequences of a null mutation in the 11-cis-retinol dehydrogenase gene in man. Vis Neurosci. 2000;17:667-678.

58. Cideciyan AV, Pugh EN Jr, Lamb TD, Huang Y, Jacobson SG. Rod plateaux during dark adaptation in Sorsby's fundus dystrophy and vitamin A deficiency. Invest Ophthalmol Vis Sci. 1997;38:17861794.

59. Steinmetz RL, Haimovici R, Jubb C, Fitzke FW, Bird AC. Symptomatic abnormalities of dark adaptation in patients with age-related Bruch's membrane change. Br J Ophthalmol. 1993;77:549-554.
60. Brown B, Adams AJ, Coletta NJ, Haegerstrom-Portnoy G. Dark adaptation in age-related maculopathy. Opbthalmic Physiol Opt. 1986;6:81-84.

61. Owsley C, Jackson GR, White M, Feist R, Edwards D. Delays in rod-mediated dark adaptation in early age-related maculopathy. Ophthalmol. 2001;108:1196-1202.

62. Bird AC. Bruch's membrane change with age. Br J Opbthalmol. 1992;763:166-188.

63. Moore DJ, Hussain AA, Marshall J. Age-related variation in the hydraulic conductivity of Bruch's membrane. Invest Ophthalmol Vis Sci. 1995;36:1290-1297.

64. Bird AC. The Bowman lecture. Towards an understanding of agerelated macular disease. Eye. 2003;17:457-466.

65. Starita C, Hussain AA, Pagliarini S, Marshall J. Hydrodynamics of ageing Bruch's membrane: implications for macular disease. Exp Eye Res. 1996;62:565-572.

66. Curcio CA, Owsley C, Jackson GR. Spare the rods, save the cones in aging and age-related maculopathy. Invest Opbthalmol Vis Sci. 2000;41:2015-2018.

67. Donoso LA, Kim D, Frost A, Callahan A, Hageman G. The role of inflammation in the pathogenesis of age-related macular degeneration. Survey Opbthalmol. 2006;51:137-152.

68. Age-Related Eye Disease Study Research Group. A randomized, placebo-controlled, clinical trial of high-dose supplementation with vitamins $\mathrm{C}$ and $\mathrm{E}$, beta carotene, and zinc for age-related macular degeneration and vision loss: AREDS report no. 8. Arch Ophthalmol. 2001;119:1417-1436.

69. McBee JK, Palczewski K, Baehr W, Pepperberg DR. Confronting complexity: the interlink of phototransduction and retinoid metabolism in the vertebrate retina. Prog Retin Eye Res. 2001;20: 469-529.

70. Thompson DA, Gal A. Vitamin A metabolism in the retinal pigment epithelium: genes, mutations and diseases. Prog Retin Eye Res. 22:683-703.

71. Baehr W, Wu SM, Bird AC, Palczewski K. The retinoid cycle and retina disease. Vision Res. 2003;43:2957-2958.

72. Yamamoto S, Sippel KC, Berson EL, Dryja TP. Defects in the rhodopsin kinase gene in the Oguchi form of stationary night blindness. Nat Genet. 1997;15:175-178.

73. Steinmetz RL, Polkinghorne PC, Fitzke FW, Kemp CM, Bird AC. Abnormal dark adaptation and rhodopsin kinetics in Sorsby's fundus dystrophy. Invest Ophthalmol Vis Sci. 1992;33:1633-1636.

74. Burstedt MSI, Sandgren O, Golovleva I, Wachtmeister L. Retinal function in Bothnia dystrophy: an electrophysiological study. $V i$ sion Res. 2003;43:2559-2571.

75. Saari JC, Nawrot M, Kennedy BN, et al. Visual cycle impairment in cellular retinaldehyde binding protein (CRALBP) knockout mice results in delayed dark adaptation. Neuron. 2001;29:739-748.

76. Sun H, Molday RS, Nathans J. Retinal stimulates ATP hydrolysis by purified and reconstituted ABCR, the photoreceptor-specific ATPbinding cassette transporter responsible for Stargardt disease. J Biol Chem. 1999;274:8269-8281.

77. Weng J, Mata NL, Azarian SM, Tzekov RT, Birch DG, Travis GH. Insights into the function of Rim protein in photoreceptors and etiology of Stargardt's disease from the phenotype in abcr knockout mice. Cell. 1999;98:13-23.

78. Sparrow JR, Fishkin N, Zhou J, et al. A2E, a byproduct of the visual cycle. Vision Res. 2003;43:2983-2990.

79. Fishman GA, Farbman JS, Alexander KR. Delayed rod dark adaptation in patients with Stargardt's disease. Opbthalmol. 1991;98: 957-962.

80. Cideciyan AV, Aleman TS, Swider M, et al. Mutations in $A B C A 4$ result in accumulation of lipofuscin before slowing of the retinoid cycle: a reappraisal of the human disease sequence. Hum Mol Gen. 2004;13:525-534.

81. Segel IH. Enzyme Kinetics: Behavior and Analysis of Rapid Equilibrium and Steady State Enzyme Systems. New York: Wiley; 1975. 